\title{
Moderate Deviations for Stochastic Fractional Heat Equation Driven by Fractional Noise
}

\author{
Xichao Sun $\mathbb{D}^{1},{ }^{1}$ Ming Li $\left(\mathbb{D},{ }^{2}\right.$ and Wei Zhao ${ }^{3}$ \\ ${ }^{1}$ College of Science, Bengbu University, 1866 Caoshan Rd., Bengbu 233030, China \\ ${ }^{2}$ School of Information Science and Technology, East China Normal University, No. 500, Dong-Chuan Road, Shanghai 200241, China \\ ${ }^{3}$ American University of Sharjah, P.O. Box 26666, Sharjah, UAE
}

Correspondence should be addressed to Xichao Sun; sunxichao626@126.com

Received 28 January 2018; Revised 6 April 2018; Accepted 2 May 2018; Published 10 July 2018

Academic Editor: Maricel Agop

Copyright (c) 2018 Xichao Sun et al. This is an open access article distributed under the Creative Commons Attribution License, which permits unrestricted use, distribution, and reproduction in any medium, provided the original work is properly cited.

We consider a class of stochastic fractional heat equations driven by fractional noises. A central limit theorem is given, and a moderate deviation principle is established.

\section{Introduction}

Since the work of Freidlin and Wentzell [1], the large deviation principle (LDP) has been extensively developed for small noise systems and other types of models (such as interacting particle systems) (see [2-7]). Cardon-Weber [2] proved a LDP for a Burgers-type SPDE driven by white noise. Marquez-Carreras and Sarra [3] proved a LDP for a stochastic heat equation with spatially correlated noise, and Mellali and Mellouk [4] extended Marquez-Carreras and Sarra's [3] to a fractional operator. Jiang et al. [5] proved a LDP for a fourth-order stochastic heat equation driven by fractional noise. Budhiraja et al. [6] studied large deviation properties of systems of weakly interacting particles. Budhiraja et al. [7] proved a large deviation for Brownian particle systems with killing.

Similar to the large deviation, the moderate deviation problems also come from the theory of statistical inference. Using the moderate deviation principle (MDP), we can get the rate of convergence and an important method to construct asymptotic confidence intervals, for example, Liming [8], Guillin and Liptser [9], Cattani and Ciancio [10], and other references therein. There are also many works about MDP about stochastic (partial) differential equations; some surveys and literatures could be found in Budhiraja et al. [11], Wang and Zhang [12], Li et al. [13], Yang and Jiang [14], and the references therein. On the other hand, fractional equations have attracted many physicists and mathematicians due to various applications in risk management, image analysis, and statistical mechanics (see Droniou and Imbert [15], Bakhoum and Toma [16], Levy and Pinchas [17], Mardani et al. [18], Niculescu et al. [19], Paun [20], and Pinchas [21] for a survey of applications). Stochastic partial differential equations involving a fractional Laplacian operator have been studied by many authors; see Mueller [22], Wu [23], Liu et al. [24], Wu [25], and the references therein.

Motived above, we investigated the moderate deviations about the stochastic fractional heat equation with fractional noise as follows:

$$
\begin{aligned}
\frac{\partial v^{\varepsilon}}{\partial t}(t, x) & =D_{\delta, \alpha} v^{\varepsilon}(t, x)+f\left(v^{\varepsilon}(t, x)\right)+\sqrt{\varepsilon} B^{H}(d t, d x), \\
v^{\varepsilon}(o, x) & =0
\end{aligned}
$$

where $t \in[0, T], x \in D=[0,1], D_{\delta, \alpha}$ is the fractional Laplacian operator which is defined in Appendix, and $B^{H}(d t, d x)$ denotes a fractional noise which is fractional in time and white in space with Hurst parameter $H \in(1 / 2,1)$; that is, $B^{H}$ is a mean zero Gaussian random field on $[0, T] \times D$ with covariance. 


$$
\operatorname{Cov}\left(B^{H}(t, x) B^{H}(s, y)\right)=\frac{1}{2}\left(t^{2 H}+s^{2 H}-|t-s|^{2 H}\right)(x \wedge y) .
$$

Assume that the coefficients satisfy the following.

Assumption 1. Function $f$ is Lipschitz; that is, there exist an $m>0$ satisfying

$$
|f(y)-f(x)| \leq m|y-x|, \quad \forall x, y \in D .
$$

Under the conditions of Assumption 1, (1) possesses a unique solution in the sense of Walsh [26] as follows:

$$
\begin{aligned}
v^{\varepsilon}(t, x)= & \int_{0}^{t} \int_{D} G_{\alpha}^{\delta}(t-s, x-z) f\left(v^{\varepsilon}(s, z)\right) d z d s \\
& +\sqrt{\varepsilon} \int_{0}^{t} \int_{D} G_{\alpha}^{\delta}(t-s, x-\mathrm{z}) B^{H}(d s, d z) .
\end{aligned}
$$

As the parameter $\varepsilon \rightarrow 0$, the solution $v^{\varepsilon}(t, x)$ of (1) will tend to $v^{0}(t, x)$ which is the solution to the following equation:

$$
\begin{aligned}
\frac{\partial v^{0}}{\partial t}(t, x) & =D_{\delta, \alpha} v^{0}(t, x)+f\left(v^{0}(t, x)\right), \\
v^{0}(0, x) & =0 .
\end{aligned}
$$

This paper mainly devotes to investigate the deviations of $v^{\varepsilon}$ from the deterministic solution $v^{0}$, as $\varepsilon \rightarrow 0$, that is, the asymptotic behavior of the trajectories.

$$
V^{\varepsilon}(t, x):=\frac{1}{\sqrt{\varepsilon} a(\varepsilon)}\left(v^{\varepsilon}-v^{0}\right)(t, x), \quad(t, x) \in[0, T] \times D,
$$

where $a(\varepsilon)$ is the same deviation scale that strongly influences the asymptotic behavior of $V^{\varepsilon}$.

If $a(\varepsilon)=1 / \sqrt{\varepsilon}$, we are in the domain of large deviation estimate, which can be proved similarly to Jiang et al. [5].

The case $a(\varepsilon) \equiv 1$ provides the central limit theorem. As $\varepsilon \downarrow 0$, we will prove that $\left(v^{\varepsilon}-v^{0}\right) / \sqrt{\varepsilon}$ converges to a random field in this paper.
To fill the gap between scale $a(\varepsilon)=1$ and scale $a(\varepsilon)=$ $1 / \sqrt{\varepsilon}$, we mainly devote to the moderate deviation when the scale satisfies the following:

$$
a(\varepsilon) \rightarrow+\infty, \quad \sqrt{\varepsilon} a(\varepsilon) \rightarrow 0, \varepsilon \rightarrow 0 .
$$

This paper is organized as follows. In Section 2, the definition of the fractional noise $B^{H}(d s, d z)$ is given. In Section 3, the main result is given and proved. In Appendix, some results about the Green kernel are given.

\section{Fractional Noise}

Let $H \in(1 / 2,1)$, and $\left\{B^{H}([0, t] \times A)\right\}_{(t, A) \in[0, T] \times \mathscr{B}(\mathbb{R})}$ is a centered Gaussian family of random variables with the covariance satisfying

$$
\mathbb{E}\left[B^{H}(t, A) B^{H}(s, B)\right]=|A \cap B| R_{H}(t, s),
$$

with $s, t \in[0, T], A, B \in \mathscr{B}(\mathbb{R})$ and covariance kernel $R_{H}$ $(t, s)=(1 / 2)\left(t^{2 H}+s^{2 H}-|t-s|^{2 H}\right)$, where $|A|$ denotes the Lebesgue measure of the set $A \in \mathscr{B}(\mathbb{R})$ and $\mathscr{B}(\mathbb{R})$ denotes the class of Borel sets in $\mathbb{R}$.

We denote $\varphi$ as the set of step functions on $[0, T] \times \mathbb{R}$. Let $\mathscr{H}$ be the Hilbert space defined as the closure of $\varphi$ with respect to the scalar product.

$$
\left\langle\mathbb{1}_{[0, t] \times A}, \mathbb{1}_{[0, s] \times B}\right\rangle_{\mathscr{H}}=\mathbb{E}\left[B^{H}(t, A) B^{H}(s, B)\right] .
$$

According to Nualart and Ouknine [27], the mapping $\mathbb{1}_{[0, t] \times A} \rightarrow B^{H}(t, A)$ can be extended to an isometry between $\mathscr{H}$ and the Gaussian space $H_{1}\left(B^{H}\right)$ associated with $B^{H}$ and denoted by

$$
\phi \mapsto B^{H}(\phi):=\int_{0}^{t} \int_{A} \phi(s, x) B^{H}(d s, d x) .
$$

Define the linear operator $K_{H}^{*}: \varphi \mapsto L^{2}([0, T])$ by

$$
K_{H}^{*}(\phi)=K_{H}(T, s) \phi(s, y)+\int_{s}^{T}(\phi(r, y)-\phi(s, y)) \frac{\partial K_{H}}{\partial r}(r, s) d r
$$

where $K_{H}$ is defined by

$$
K_{H}(t, s):=\left\{\begin{array}{l}
C_{H} s^{(1 / 2)-H} \int_{s}^{t}(u-s)^{H-(3 / 2)}\left(1-\left(\frac{s}{u}\right)^{(1 / 2)-H}\right) d u+C_{H}(t-s)^{H-(1 / 2)}, \quad 0<s \leq t \\
0, \quad \text { otherwise, }
\end{array}\right.
$$

with $C_{H}=(2 \alpha \Gamma((3 / 2)-\alpha) / \Gamma(\alpha+(1 / 2)) \Gamma(2-2 \alpha))^{1 / 2}, \quad$ and one can get

$$
\frac{\partial K_{H}}{\partial t}(t, s)=C_{H}\left(\frac{1}{2}-H\right)(t-s)^{H-(3 / 2)}\left(\begin{array}{c}
t \\
s
\end{array}\right)^{H-(1 / 2)}
$$

Moreover, $K_{H}$ satisfies the following:

$$
\int_{0}^{s \wedge t} K_{H}(s, r) K_{H}(t, r) d r=\frac{1}{2}\left[t^{2 H}+s^{2 H}-|t-s|^{2 H}\right] .
$$


Then, since

$$
\left(K_{H}^{*} 1_{[0, t] \times A}\right)(s, x)=K_{H}(t, s) 1_{[0, t] \times A}(s, x),
$$

one can get

$$
\left\langle K_{H}^{*}(\phi), K_{H}^{*}(\psi)\right\rangle_{L^{2}([0, T] \times D)}=\langle\phi, \psi\rangle \mathscr{H},
$$

where $\phi$ and $\psi$ in $\varphi$ are any step functions. So the operator $K_{H}^{*}$ gives an isometry between the Hilbert space $\mathscr{H}$ and $L^{2}([0, T] \times[0,1])$. Hence, $\{W(t, A), t \in[0, T], A \in$ $\mathscr{B}[0,1]\}$ defined by

$$
W(t, A)=B^{H}\left(\left(K_{H}^{*}\right)^{-1}\left(1_{[0, t] \times A}\right)\right)
$$

is a space-time white noise, and $B^{H}$ has the following form:

$$
B^{H}(t, x)=\int_{0}^{t} \int_{0}^{x} K_{H}(t, s) W(d s, d y) .
$$

Therefore, the mild formulation of (4) has the following form:

$$
\begin{aligned}
v^{\varepsilon}(t, x)= & \int_{0}^{t} \int_{D} G_{\alpha}^{\delta}(t-s, x-z) f\left(v^{\varepsilon}(s, z)\right) d z d s \\
& +\sqrt{\varepsilon} \int_{0}^{t} \int_{D} K_{H}^{*} G_{\alpha}^{\delta}(t-s, x-z) W(d s, d z) .
\end{aligned}
$$

That is, the last term of (4) is equal to

$$
\begin{aligned}
& \int_{0}^{t} \int_{D} G_{\alpha}^{\delta}(-s, x-z) B^{H}(d s, d z) \\
&=\int_{0}^{t} \int_{D} K_{H}^{*} G_{\alpha}^{\delta}(t-s, x-z) W(d s, d z) .
\end{aligned}
$$

The following embedding proposition is given by Nualart and Ouknine [27].

Lemma 1. Set $H>(1 / 2)$, then, we have

$$
L^{1 / H}([0, T] \times \mathbb{R}) \subset \mathscr{H} .
$$

\section{Main Results and Their Proof}

3.1. Main Results. For any function $\phi$ defined on $[0, T] \times D$, let

$$
\begin{aligned}
\|\phi\|_{t, \infty} & =\sup \{|\phi(s, z)|:(s, z) \in[0, t] \times D\}, \\
\|\phi\|_{t, \gamma} & =\sup \left\{\frac{\left|\phi\left(s_{1}, z_{1}\right)-\phi\left(s_{2}, z_{2}\right)\right|}{\left(\left|s_{1}-s_{2}\right|^{\mu}+\left|z_{1}-z_{2}\right|^{\theta}\right)^{\gamma}}:\left(s_{1}, z_{1}\right) \neq\left(s_{2}, z_{2}\right) \in[0, t] \times D\right\},
\end{aligned}
$$

where $\mu \in(0,(\alpha+1) H-1 / \alpha), \theta \in(0, \min \{1,(\alpha+1) H-1\})$, and $\gamma \in(0,1)$. Let

$$
\|\phi\|_{\gamma}=|\phi|_{T, \infty}+\|\phi\|_{T, \gamma} .
$$

Let $\mathscr{C}^{\gamma}([0, T] \times D)$ be the functions $\phi:[0, T] \times D$ which satisfy $\|\phi\|_{\gamma}<\infty$, endowed with the $\|\phi\|_{\gamma}$ - norm.

Define

$$
\mathscr{E}=\left\{e(x, t)=\int_{0}^{t} \int_{0}^{x} \bar{e}(s, z) d s d z, \quad \bar{e} \in L^{2}([0, T] \times D)\right\},
$$

which is a Cameron-Martin space endowed with the norm

$$
\|e\|_{\mathscr{E}}=\left(\int_{0}^{t} \int_{0}^{x} \bar{e}^{2}(s, z) d z d s\right)^{1 / 2} .
$$

Suppose $e \in \mathscr{E}$. Now, let $v^{e}$ be the solution of the following deterministic equation:

$$
\begin{aligned}
\frac{\partial v^{e}}{\partial t}(t, x) & =D_{\delta, \alpha} v^{e}(t, x)+f\left(v^{e}(t, x)\right)+F(\bar{e}(t, x)), \\
v^{e}(0, x) & =0,
\end{aligned}
$$

where

$$
F(\bar{e}(x, t)):=\frac{\partial^{2}}{\partial x \partial t} \int_{0}^{t} \int_{0}^{x} K_{H}(t, s) \bar{e}(z, s) d z d s
$$

satisfies the following:

$$
\begin{aligned}
& \int_{0}^{t} \int_{D} G_{\alpha}^{\delta}(t-s, x-z) F(\bar{e}(z, e)) d z d s \\
&=\int_{0}^{t} \int_{D} K_{H}^{*} G_{\alpha}^{\delta}(t-s, x-z) \bar{e}(z, s) d z d s .
\end{aligned}
$$

The above (26) shows a unique mild solution.

$$
\begin{aligned}
Z^{e}(x, t)= & \int_{0}^{t} \int_{D} G_{\alpha}^{\delta}(t-s, x-z) f\left(s, z, Z^{e}(z, s)\right) d z d s \\
& +\int_{0}^{t} \int_{D} K_{H}^{*} G_{\alpha}^{\delta}(t-s, x-z) \bar{e}(z, s) d z d s
\end{aligned}
$$

Similar to Jiang et al. [5], one can get the following: 
Theorem 1. Let $H \in(1 / 2,1)$. Under Assumption1, the law of the solution to (1) satisfies a deviation principle on $\mathscr{C}^{\gamma}(D \times[0, T])$ with the good rate function:

$$
I(\varphi)=\inf \left\{\frac{1}{2}\|e\|_{\mathscr{E}}^{2} ; Z^{e}=\varphi\right\}
$$

with the convention inf $\varnothing=\infty$, where $0<\gamma<1$.

More precisely, for any Borel measurable subset $B$ of $\mathscr{C}^{\gamma}([0, T] \times D)$,

$$
\begin{aligned}
-\inf _{\varphi \in B^{o}} I(\varphi) & \leq \liminf _{\varepsilon \rightarrow 0} \varepsilon \log P\left(Z^{e} \in B\right) \\
& \leq \lim _{\varepsilon \rightarrow 0} \sup \varepsilon \log P\left(Z^{e} \in B\right) \leq-\inf _{\varphi \in \bar{B}} I(\varphi),
\end{aligned}
$$

where $B^{o}$ and $\bar{B}$ denote the interior and the closure of $B$, respectively.

We furthermore suppose that the coefficients satisfy the following.

Assumption 2. $f$ is differentiable, and the derivative $f^{\prime}$ of $f$ is Lipschitz. That is to say, there exist positive constant $m$ and $m^{\prime}$ which satisfy the following:

$$
\left|f^{\prime}(y)-f^{\prime}(x)\right| \leq m^{\prime}|y-x|, \quad \forall x, y \in D .
$$

Together with the Lipschitz of $f$, we conclude that

$$
\left|f^{\prime}(x)\right| \leq m \text {. }
$$

Now, we give the following central limit theorem.

Theorem 2. Let $f$ and its derivative $f^{\prime}$ satisfy Assumptions 1 and 2. Then, for $p \geq 1,\left(v^{e}-v^{0}\right) / \sqrt{\varepsilon}$ converges in $L^{p}$ to a random field $U$ on $\mathscr{C}^{\gamma}([0, T] \times D)$ with $0<\gamma<1$, determined by

$$
\begin{aligned}
\frac{\partial U(t, x)}{\partial t} & =D_{\delta, \alpha} U(t, x)+f^{\prime}\left(v^{0}(t, x)\right) U(t, x)+B^{H}(d t, d x), \\
U(0, x) & =0,
\end{aligned}
$$

for all, $(t, x) \in[0, T] \times D$.

Let the function $U^{e}$ be the solution to the following partial differential equation:

$$
\begin{aligned}
\frac{\partial U^{e}(t, x)}{\partial t} & =D_{\delta, \alpha} U^{e}(t, x)+f^{\prime}\left(v^{0}(t, x)\right) U^{e}(t, x)+F(\bar{e}(t, x)) \\
U^{e}(0, x) & =0 .
\end{aligned}
$$

Under Assumptions 1 and 2, by Theorem 1, one can get $U / a(\varepsilon)$ which satisfies large deviation principles on $\mathscr{C}^{\gamma}([0, T] \times D)$ with the speed $e^{2}(\varepsilon)$ and the good rate function satisfies the following:

$$
I(\varphi)\left\{\begin{array}{l}
\inf \left\{\frac{1}{2}\|e\|_{\mathscr{E}}^{2}, \quad U^{e}=\varphi\right\} \\
+\infty, \quad \text { otherwise. }
\end{array}\right.
$$

Now, the second result is given as follows:

Theorem 3. In moderate deviation principle, let $H \in(1 / 2,1)$. Under the Assumptions1and2, then, the random field (1/ $\sqrt{\varepsilon} a(\varepsilon))\left(v^{\varepsilon}-v^{0}\right)$ satisfies a large deviation principle on the space $\mathscr{C}^{\gamma}([0, T] \times D)$ with speed $a^{2}(\varepsilon)$ and the good rate function $I(\varphi)$ defined by (36), where $0<\gamma<1$.

\subsection{Convergence of the Solution}

Lemma 2. Let $H \in(1 / 2,1)$. Under Assumption1, then, there exists a unique solution to (1). Moreover, for any $p \in[1, \infty), T>0$,

$$
\sup _{\varepsilon \geq 1} \sup _{(t, x) \in[0, T] \times D} E\left|v^{\varepsilon}(t, x)\right|^{p}<\infty \text {. }
$$

As $\varepsilon \rightarrow 0$, we get the convergence of $v^{\varepsilon}$ as follows:

Proposition 1. Let $H \in(1 / 2,1)$ and $p \geq 2$. By Assumption 1, there exists a constant $c(m, p, H, T)$ which satisfies the following:

$$
E\left(\left|v^{\varepsilon}-v^{0}\right|_{t, \infty}\right)^{p} \leq \varepsilon^{p / 2} c(m, p, H, T) .
$$

Proof. Note that

$$
\begin{gathered}
v^{\varepsilon}(t, x)-v^{0}(t, x) \\
=\int_{0}^{t} \int_{D} G_{\alpha}^{\delta}(t-s, x-z)\left[f\left(v^{\varepsilon}(s, z)\right)-f\left(v^{0}(s, z)\right)\right] d z d s \\
\quad+\sqrt{\varepsilon} \int_{0}^{t} \int_{D} G_{\alpha}^{\delta}(t-s, x-z) B^{H}(d s d z) .
\end{gathered}
$$

One can get

$$
\begin{aligned}
\left(\left|v^{\varepsilon}(t, x)-v^{0}(t, x)\right|_{T, \infty}\right)^{p} \leq & 2^{p-1}\left(\sup _{(t, x) \in[0, T] \times D}\left|\int_{0}^{t} \int_{D} G_{\alpha}^{\delta}(t-s, x-z)\left[f\left(v^{\varepsilon}(s, z)\right)-f\left(v^{0}(s, z)\right)\right] d z d s\right|\right)^{p} \\
& +\varepsilon^{p / 2} 2^{p-1}\left(\sup _{(t, x) \in[0, T] \times D}\left|\int_{0}^{t} \int_{D} G_{\alpha}^{\delta}(t-s, x-z) B^{H}(d s d z)\right|\right)^{p} .
\end{aligned}
$$


Set

$$
\begin{aligned}
& N_{1}^{\varepsilon}(t, x)=\int_{0}^{t} \int_{D} G_{\alpha}^{\delta}(t-s, x-z)\left[f\left(v^{\varepsilon}(s, z)\right)-f\left(v^{0}(s, z)\right)\right] d z d s, \\
& N_{2}^{\varepsilon}(t, x)=\int_{0}^{t} \int_{D} G_{\alpha}^{\delta}(t-s, x-z) B^{H}(d s d z) .
\end{aligned}
$$

Together with Hölder's inequality, the Lipschitz condition (C) and (5) of Lemma A.1, for $1 / \alpha<q<\alpha$, we have

$$
\begin{aligned}
E\left(\left|N_{1}^{\varepsilon}\right|_{T, \infty}\right)^{p} \leq & m^{p}\left(\sup _{(t, x) \in[0, T] \times D} G_{\alpha}^{\delta}(t-s, x-z)^{q} d z d s\right)^{p / q} \\
& \times E \int_{0}^{T}\left(\left|v^{\varepsilon}-v^{0}\right|_{T, \infty}\right)^{p} d t \\
\leq & C(p, m, T) E \int_{0}^{T}\left(\left|v^{\varepsilon}-v^{0}\right|_{T, \infty}\right)^{p} d t
\end{aligned}
$$

where $(1 / p)+(1 / q)=1$.
For any $0 \leq t \leq T, x, z \in D, p>2$, by (21) and (A.9), there exist $\theta \in(0, \min \{1,(\alpha+1) H-1\})$ which satisfies the following:

$$
\begin{aligned}
& E\left|N_{2}^{\varepsilon}(t, x)-N_{2}^{\varepsilon}\left(t, x^{\prime}\right)\right|^{p} \\
& \left.\quad=E \mid \int_{0}^{t} \int_{D}\left[G_{\alpha}^{\delta}(t-s, x-z)-G_{\alpha}^{\delta}\left(t-s, x^{\prime}-z\right)\right] B^{H}(d s d z)\right]\left.\right|^{p} \\
& \quad \leq\left|\int_{0}^{t} \int_{D}\left[K_{H}^{*}\left(G_{\alpha}^{\delta}(t-s, x-z)-G_{\alpha}^{\delta}\left(t-s, x^{\prime}-z\right)\right)\right]^{2} d s d z\right|^{p / 2} \\
& \left.\quad \leq\left.\left(\int_{0}^{t} \int_{D} \mid G_{\alpha}^{\delta}(t-s, x-z)-G_{\alpha}^{\delta}\left(t-s, x^{\prime}-z\right)\right)\right|^{1 / H} d s d z\right)^{H p} \\
& \quad \leq m\left|x-x^{\prime}\right|^{p \theta} .
\end{aligned}
$$

Similarly,

$$
\begin{aligned}
E\left|N_{2}^{\varepsilon}(t, x)-N_{2}^{\varepsilon}\left(t^{\prime}, x\right)\right|^{p} & \left.=E \mid \int_{0}^{t} \int_{D}\left[G_{\alpha}^{\delta}(t-s, x-z)-G_{\alpha}^{\delta}\left(t^{\prime}-s, x-z\right)\right] B^{H}(d s d z)\right]\left.\right|^{p} \\
\leq & \left|\int_{0}^{t} \int_{D}\left[K_{H}^{*}\left(G_{\alpha}^{\delta}(t-s, x-z)-G_{\alpha}^{\delta}\left(t^{\prime}-s, x-z\right)\right)\right]^{2} d s d z\right|^{p / 2} \\
\leq & C(p, m, T)\left|\int_{t^{\prime}}^{t} \int_{D}\left[K_{H}^{*}\left(G_{\alpha}^{\delta}(t-s, x-z)\right)\right]^{2} d s d z\right|^{p / 2} \\
& +C(p, m, T)\left|\int_{0}^{t^{\prime}} \int_{D}\left[K_{H}^{*}\left(G_{\alpha}^{\delta}(t-s, x-z)-G_{\alpha}^{\delta}\left(t^{\prime}-s, x-z\right)\right)\right]^{2} d s d z\right|^{p / 2} .
\end{aligned}
$$

By (21), (A.10), and (A.11), for $\mu \in(0,((\alpha+1) H-1) / \alpha)$, one can get

$$
\begin{gathered}
\left|\int_{t^{\prime}}^{t} \int_{D}\left[K_{H}^{*}\left(G_{\alpha}^{\delta}(t-s, x-z)\right)\right]^{2} d s d z\right|^{p / 2} \\
\leq C_{H}\left(\int_{t^{\prime}}^{t} \int_{D}\left|G_{\alpha}^{\delta}(t-s, x-z)\right|^{1 / H} d s d z\right)^{H p} \\
\leq C_{H}\left(t-t^{\prime}\right) p(((\alpha+1) H-1) / \alpha)
\end{gathered}
$$

and

$$
\begin{aligned}
& \left|\int_{0}^{t^{\prime}} \int_{D}\left[K_{H}^{*}\left(G_{\alpha}^{\delta}(t-s, x-z)-G_{\alpha}^{\delta}\left(t^{\prime}-s, x-z\right)\right)\right]^{2} d s d z\right|^{p / 2} \\
& \quad \leq C_{H}\left(\int_{t^{\prime}}^{t} \int_{D}\left|K_{H}^{*}\left(G_{\alpha}^{\delta}(t-s, x-z)-G_{\alpha}^{\delta}\left(t^{\prime}-s, x-z\right)\right)\right|^{1 / H} d s d z\right)^{H p} \\
& \quad \leq C_{H}\left(t-t^{\prime}\right)^{p \mu}
\end{aligned}
$$

Together with (43), (44), (45), and (46), one can get for any $p \geq 2$ and $\left(t^{\prime}, x^{\prime}\right),(t, x) \in[0, T] \times D$, there exists a constant $\beta>0$ such that

$$
E\left|N_{2}^{\varepsilon}(t, x)-N_{2}^{\varepsilon}\left(t, x^{\prime}\right)\right|^{p} \leq C(p, m, H, T)\left(\left|x-x^{\prime}\right|^{\theta}+\left|t-t^{\prime}\right|^{\mu}\right)^{p \beta},
$$

where $C(p, m, H, T)$ is independent of $\varepsilon$. For $p>2 / \beta$, by Garsis-Rodemich-Rumser's Lemma, there exist a constant $C$ and a random variable $M_{p, \varepsilon}(\omega)$ satisfying

$$
\begin{aligned}
\mid N_{2}^{\varepsilon}(t, x)- & \left.N_{2}^{\varepsilon}\left(t, x^{\prime}\right)\right|^{p} \\
\leq & m_{p, \varepsilon}(\omega)\left(\left|x-x^{\prime}\right|^{\theta}+\left|t-t^{\prime}\right|^{\mu}\right)^{p \beta-2} \\
& \cdot\left(\log \left(\frac{c}{\left|x-x^{\prime}\right|^{\theta}+\left|t-t^{\prime}\right| \mu}\right)\right)^{2}
\end{aligned}
$$


and

$$
\sup _{\varepsilon \in(0,1)} E\left[m_{p, \varepsilon}\right]<\infty
$$

If we choose $t^{\prime}=0$ in (48), we can get

$$
\begin{gathered}
E\left(\sup _{(t, x) \in[0, T] \times D} \int_{0}^{t} \int_{D} G_{\alpha}^{\delta}(t-s, x-z) B^{H}(d s d z)\right)^{p} \\
\leq c(m, p, H, T) \sup _{\varepsilon \in(0,1)} E\left[m_{p, \varepsilon}\right]<\infty .
\end{gathered}
$$

By Gronwall's inequality and (40), (42), and (48), there exists a constant $C(p, m, H, T)$ satisfying

$$
E\left(\left|v^{\varepsilon}-v^{0}\right|_{t, \infty}\right)^{p} \leq \varepsilon^{p / 2} c(m, p, H, T)
$$

The proof is completed.

3.3. The Proof of the Main Results. We first prove Theorem 1. Our proof is based on the following proposition (see Doss and Priouret [28]).

Proposition 2. Suppose $\left\{X_{j}^{\epsilon}, \epsilon>0\right\}$ and $(j=1,2)$ are two families of random variables and $\left(E_{j}, d_{j}\right)$ and $(j=1,2)$ are two Polish spaces. Suppose that
(1) There exists a map $K:\left\{I_{1}<\infty\right\} \rightarrow E_{1}$ such that $\left\{I_{1} \leq a\right\} \rightarrow E_{1}$ is continuous for any $a<\infty$.

(2) $\left\{X_{1}^{\epsilon}, \epsilon>0\right\}$ satisfies with the rate functions $I_{1}: E_{1} \rightarrow$ $[0, \infty]$.

(3) For any $R, \delta, a>0$, there exist $\rho>0$ and $\epsilon_{0}>0$ satisfying $I_{1} \leq a$ and $\epsilon \leq \epsilon_{0}$ for all $h \in E_{1}$, and

$$
P\left(d_{1}\left(X_{1}^{\epsilon}\right) \leq \rho, d_{2}\left(x_{2}^{\epsilon}, K(e)\right) \geq \delta\right) \leq \exp \left(-\frac{R}{\epsilon^{2}}\right)
$$

Then, $\left\{X_{2}^{\epsilon}, \epsilon>0\right\}$ satisfies a LDP with the rate functions

$$
I(h)=\inf \left\{I_{1}(e): K(e)=h\right\}
$$

To prove Theorem 1, one only needs to prove

(i) Under some topology, $Z(e):\left\{e:\|e\|_{\mathscr{E}} \leq a\right\} \rightarrow$ $C\left([0, T], L^{p}(D)\right)$ is continuous for any $a>0$.

(ii) In Freidin-Wentzell inequality, for any $R>0, \eta>0$, and $e \in \mathscr{E}$, there exist a $\delta>0$ satisfying

$$
\lim _{\epsilon \rightarrow 0} \sup \epsilon^{2} \log P\left(\|\epsilon W-e\|_{\infty}<\delta, \sup _{t \in[0, T]} u^{\epsilon}(t, \cdot)-Z(e)(t, \cdot) \|_{2} \geq \rho\right) \leq-R
$$

Theorem 4. When the level set $\left\{\|e\|_{\mathscr{E}} \leq a\right\}$ is endowed with the topology of uniform convergence on $[0, T] \times D$,

$$
Z(e):\left\{e:\|e\|_{\mathscr{E}} \leq a\right\} \rightarrow C\left([0, T], L^{p}(D)\right)
$$

is the continuous map for any $a \in[0,+\infty)$.
Proof. One only needs to prove that for fixed $a>0, e, h \in$ $\left\{\|e\|_{\mathscr{E}} \leq a\right\}$,

$$
\sup _{t \in[0, T]} \int_{\mathbb{R}}|Z(e)(t, x)-Z(h)(t, x)|^{2} d x \leq C|| e-h \|_{\mathscr{E}} .
$$

Note that

$$
\begin{aligned}
Z(e)(t, x)-Z(h)(t, x)= & \int_{0}^{t} \int_{\mathbb{R}} G_{\alpha}^{\delta}(t-s, x-z)(f(s, z, Z(e)(s, z))-f(s, z, Z(h)(s, z))) d z d s \\
& +\int_{0}^{t} \int_{\mathbb{R}} K_{H}^{*} G_{\alpha}^{\delta}(t-s, x-z) \bar{e}(s, z) d z d s:=\mathrm{I}(t, x)+\operatorname{II}(t, x) .
\end{aligned}
$$

Using (A.5) in Appendix with $p=q=2$, then $\rho=1$, one can get

$$
\|\mathrm{I}(t, x)\|_{2}^{2} \leq C \int_{0}^{t}(t-s)^{-(1 / \alpha)+(1 / \alpha \rho)} \|\left(f(s, z, Z(e)(s, z))-f(s, z, Z(h)(s, z))\left\|_{2} d s \leq C \sup _{0 \leq s \leq t}\right\| Z(e)(s, \cdot)-Z(h)(s, \cdot) \|_{2} .\right.
$$


Now, we deal with $\operatorname{II}(t, x)$, together with

$$
\begin{aligned}
\|\mathrm{II}(t, x)\|_{2}^{2} & =\int_{\mathbb{R}}\left|\int_{0}^{t} \int_{\mathbb{R}} K_{H}^{*} G_{\alpha}^{\delta}(t-s, x-z)(\bar{e}(s, z)-\bar{h}(s, z)) d z d s\right|^{2} d x \\
& \leq C \int_{\mathbb{R}} \int_{0}^{t} \int_{\mathbb{R}}\left|K_{H}^{*} G_{\alpha}^{\delta}(t-s, x-z)\right|^{2} d z d s d x \times\|e-h\|_{E}^{2}:=C M(t) \times\|e-h\|_{\mathscr{E}}^{2} .
\end{aligned}
$$

Recalling $K_{H}^{*}$ is defined by (11), one can get

$$
\begin{aligned}
\left(K_{H}^{*} \phi\right)(s, x) & \leq \max _{w \in[s, T]} \phi(w, x) K_{H}(T, s), \\
M(t) & =\int_{\mathbb{R}} \int_{0}^{t} \int_{\mathbb{R}}\left|K_{H}^{*} G_{\alpha}^{\delta}(t-s, x-z)\right|^{2} d z d s d x \\
& \leq \int_{\mathbb{R}} \int_{0}^{t} \int_{\mathbb{R}}\left|\max _{w \in[s, t]} G_{\alpha}^{\delta}(t-s, x-z)\right|^{2} K_{H}^{2}(t, s) d z d x d s \\
& \leq C \int_{0}^{t}(t-s)^{-(1 / \alpha)}(t-s)^{2 H-1} d s<\infty .
\end{aligned}
$$

Using Gronwall's inequality, we can get

$$
\sup _{t \in[0, T]} \int_{\mathbb{R}}|Z(e)(t, x)-Z(h)(t, x)|^{2} d x \leq C\|e-h\|_{\mathscr{C}}
$$

The proof of the theorem is completed.

We now prove the Freidin-Wentizell inequality as follows:
Suppose $\{W(t, x),(t, x) \in[0, T] \times \mathbb{R}\}$ is a Brownian sheet. For $e \in \mathscr{E}$ and $\epsilon>0$, we define

$$
\tilde{W}(t, x)=W(t, x)-\frac{e(x, t)}{\epsilon}
$$

and

$$
\frac{\tilde{d} p}{d p}=\exp \left(\frac{1}{\epsilon} \int_{0}^{T} \int_{\mathbb{R}} \bar{e}(t, x) W(d t, d x)-\frac{1}{2 \epsilon^{2}} \int_{0}^{T} \int_{\mathbb{R}} \bar{e}^{2}(t, x) d x d t\right):=Z_{T} .
$$

Using Girsanov's theorem, the process $\tilde{W}$ is a Brownian sheet under $\tilde{P}$. Suppose $\tilde{v}^{\varepsilon}(t, x)$ is a solution of (1) under $\tilde{p}$. Then,

$$
\begin{aligned}
\tilde{v}^{\epsilon}(t, x)= & \int_{\mathbb{R}} G_{\alpha}^{\delta}(t, x-z) v_{0}(z) d z \\
& +\int_{0}^{t} \int_{\mathbb{R}} G_{\alpha}^{\delta}(t-s, x-z) f\left(t, x, \tilde{v}^{\varepsilon}(s, z)\right) d z \mathrm{~d} s \\
& +\int_{0}^{t} \int_{\mathbb{R}} K_{H}^{*} G_{\alpha}^{\delta}(t-s, x-z) \bar{e}(s, z) d z d s \\
& +\epsilon \int_{0}^{t} \int_{\mathbb{R}} K_{H}^{*} G_{\alpha}^{\delta}(t-s, x-z) \tilde{W}(d s, d z) .
\end{aligned}
$$

Now, one can prove (36). Note that, under $\tilde{p}$, then,

$$
\begin{aligned}
\left\|\tilde{u}^{\epsilon}(t, \cdot)-Z(e)(t, \cdot)\right\|_{2}= & \left\|\int_{0}^{t} \int_{\mathbb{R}} G_{\alpha}^{\delta}(t-s, \cdot-z)\left(f\left(t, x, \tilde{u}^{\varepsilon}(s, z)\right)-f(t, x, Z(e)(s, z))\right) d z d s\right\|_{2} \\
& +\left\|\epsilon \int_{0}^{t} \int_{\mathbb{R}} K_{H}^{*} G_{\alpha}^{\delta}(t-s, \cdot-y) \tilde{W}(d s, d z)\right\|_{2} \\
\leq & \int_{0}^{t} \int_{\mathbb{R}}\left\|\tilde{u}^{\varepsilon}(s, \cdot)-Z(e)(s, \cdot)\right\|_{2} d z d s+\left\|\epsilon \int_{0}^{t} \int_{\mathbb{R}} K_{H}^{*} G_{\alpha}^{\delta}(t-s, \cdot-z) \tilde{W}(d s, d z)\right\|_{2}
\end{aligned}
$$

So under $\tilde{p}$, by Gronwall's Lemma, one can get

$$
\sup _{t \in[0, T]}\left\|\tilde{u}^{\varepsilon}(s, \cdot)-Z(h)(s, \cdot)\right\|_{2} \leq C \sup _{t \in[0, T]}\left\|\epsilon \int_{0}^{t} \int_{\mathbb{R}} K_{H}^{*} G_{\alpha}^{\delta}(t-s, x-z) \tilde{W}(d s, d z)\right\|_{2} .
$$


Now, one can change (34) the proof to the following theorem.
Theorem 5. Suppose $e \in \mathscr{E}$ and $e \in \mathscr{E} \leq a$. For each $R>0$, $\eta>0$, and $e \in \mathscr{E}$, there exists a constant $\delta>0$ satisfying

$$
\lim _{\epsilon \rightarrow 0} \sup \epsilon^{2} \log \tilde{P}\left(\sup _{t \in[0, T]}\left\|\epsilon \int_{0}^{t} \int_{\mathbb{R}} K_{H}^{*} G_{\alpha}^{\delta}(t-s, x-z) \tilde{W}(d s, d z)\right\|_{2} \geq \rho,\|\epsilon \tilde{W}\|_{\infty}<\delta\right) \leq-R
$$

In the following, we give a key Lemma to prove Theorem 1, which is similar to Candon-Weber [2], and the proof is omitted.

Lemma 3. Suppose $F:([0, T] \times D)^{2} \rightarrow \mathbb{R}, C_{F}>0$ and $\beta_{0}>0$ satisfying

$$
\int_{0}^{T} \int_{D}|F(t, x, v, z)-F(s, y, v, z)|^{2} d u d z \leq C_{F}\left(|t-s|^{\mu}+|x-y|^{\theta}\right)^{\beta 0}
$$

for any $(s, z)$ and $(t, x) \in[0, T] \times D$. Suppose $N: \Omega \times$ $[0, T] \times[0,1] \rightarrow \mathbb{R}$ which is an almost surely continuous, $\mathscr{F}_{t}$-adapted process satisfying sup $\{|N(t, x)|:(t, x) \in[0, T] \times$ $D\} \leq \rho, a, s$, and for $(t, x) \in[0, T] \times D$, suppose

$$
\mathfrak{F}(t, x)=\int_{0}^{T} \int_{D} F(t, x, v, z) N(v, z) W(d v d z) .
$$

Then, for any $0<\beta<\beta_{0} / 2$, there exist a positive constant $C, C\left(\beta, \beta_{0}\right)$, and $C\left(\beta, \beta_{0}\right)$ such that for all $M \geq \rho C_{F}^{1 / 2} C\left(\beta, \beta_{0}\right)$ $C\left(\beta, \beta_{0}\right)$,

$$
P\left(\|\mathfrak{F}\|_{t, \beta} \geq M\right) \leq C \exp \left(-\frac{M^{2}}{\left.\rho^{2} C_{z} C \beta, \beta_{0}\right) C^{2}\left(\beta, \beta_{0}\right)}\right)
$$

Proof of Theorem 5. Suppose

$$
F(t, x, v, z)=\epsilon K_{H}^{*} G_{\alpha}^{\delta}(t-v, x-z) .
$$

$$
\begin{aligned}
\int_{0}^{t} \int_{D} \mid K_{H}^{*} & \left.\left(G_{\alpha}^{\delta}(t-v, x-z)-G_{\alpha}^{\delta}(s-v, y-z)\right)\right|^{2} d v d z \\
& \leq C\left(\int_{0}^{t} \int_{\mathbb{R}}\left|K_{H}^{*}\left(G_{\alpha}^{\delta}(t-v, x-z)-G_{\alpha}^{\delta}(s-v, x-z)\right)\right|^{2} d v d z+\int_{0}^{t} \int_{\mathbb{R}}\left|K_{H}^{*}\left(G_{\alpha}^{\delta}(s-v, x-z)-G_{\alpha}^{\delta}(s-v, y-z)\right)\right|^{2} d v d s\right) \\
& \leq C\left(\left(\int_{o}^{t} \int_{\mathbb{R}}\left|G_{\alpha}^{\delta}(t-v, x-z)-G_{\alpha}^{\delta}(s-v, x-z)\right|^{1 / H} d v d s\right)^{2 H}+\left(\int_{0}^{t} \int_{\mathbb{R}}\left|G_{\alpha}^{\delta}(s-v, x-z)-G_{\alpha}^{\delta}(s-v, y-z)\right|^{1 / H} d v d z\right)^{2 H}\right) \\
& \leq C\left(|t-s|^{\mu}+|x-z| \theta\right)^{\beta 0} .
\end{aligned}
$$

If $R \leq\left(\rho^{2} / 2 C_{F}\right) C^{2}(V)$ and $\rho \leq K(v) C(v) \sqrt{C_{F}}$, by Lemma 2 , we can get

$$
\begin{gathered}
\tilde{P}\left(\sup _{t \in[0, T]}\left\|\epsilon \int_{0}^{t} \int_{\mathbb{R}} K_{H}^{*} G_{\alpha}^{\delta}(t-s, x-z) \tilde{W}(d s, d z)\right\|_{2} \geq \rho\right) \\
\leq\left(\sqrt{(2)} T^{2} \pi^{2}+1\right) \exp \left(-\frac{R}{\epsilon^{2}}\right) .
\end{gathered}
$$

The proof of Theorem 3 is completed.

In the following, we first give Garsis-Rodemich-Rumser's Lemma in Bally et al. [29].
Lemma 4. Assume $p>1$ and $\left\{U^{\varepsilon}(t, x):(t, x) \in[0, T] \times D\right\}$ are a family of real-valued stochastic processes. Suppose the following is true.

Assumption 3. One has

$$
\lim _{\varepsilon \rightarrow 0} E\left|U^{\mathcal{E}}(t, x)\right|^{p}=0
$$

as $(t, x) \in[0, T] \times D$ 
Assumption 4. For any $(t, x),(s, z) \in[0, T] \times D$, there exists $v>0$ satisfying

$$
E\left|U^{\varepsilon}(t, x)-U^{\varepsilon}(s, z)\right|^{p} \leq C\left(|t-s|+|x-z|^{2}\right)^{\gamma} .
$$

Then, for any $r \in[1, p), \gamma \in(0, \beta / p)$,

$$
\lim _{\varepsilon \rightarrow 0} E\left(\left\|U^{\varepsilon}\right\|_{\gamma}\right)^{r}=0
$$

Now we can prove Theorem 2.

Proof of Theorem 2. Set $U^{\varepsilon}=\left(v^{\varepsilon}-v^{0}\right) / \sqrt{\varepsilon}$. We will prove that

$$
\lim _{\varepsilon \rightarrow 0} E\left(\left\|U^{\varepsilon}-U\right\|_{\gamma}\right)^{r}=0
$$

To this end, we need to prove that Assumptions 3 and 4 are satisfied for $W^{\varepsilon}=U^{\varepsilon}-U$. Note that

$$
\begin{aligned}
& U^{\varepsilon}(t, x)-U(t, x) \\
& =\int_{0}^{t} \int_{D} G_{\alpha}^{\delta}(t-s, x-z) \\
& \quad \cdot\left(\frac{f\left(v^{\varepsilon}(s, z)\right)-f\left(v^{0}(s, z)\right)}{\sqrt{\varepsilon}}-f^{\prime}\left(v^{0}(s, z)\right) U(s, z)\right) d s d z \\
& =: \operatorname{III}^{\varepsilon}(t, x)+\operatorname{IV}^{\varepsilon}(t, x)
\end{aligned}
$$

where

$\operatorname{III}^{\varepsilon}(t, x)$

$$
\begin{aligned}
=\int_{0}^{t} \int_{D} G_{\alpha}^{\delta}(t-s, x-z) \\
\quad \cdot\left(\frac{f\left(v^{\varepsilon}(s, z)\right)-f\left(v^{0}(s, z)\right)}{\sqrt{\varepsilon}}-f^{\prime}\left(v^{0}(s, z)\right) U^{\varepsilon}(s, z)\right) d s d z
\end{aligned}
$$

and

$$
\operatorname{IV}^{\varepsilon}(t, x)=\int_{0}^{t} \int_{D} G_{\alpha}^{\delta}(t-s, x-z) f^{\prime}\left(v^{0}(s, z)\right)\left(U^{\varepsilon}(s, z)-U(s, z)\right) d s d z .
$$

Using Taylor's formula, there exists a $\xi^{\mathcal{\varepsilon}}(t, x)$ such that

$$
\begin{aligned}
f\left(v^{\varepsilon}(t, x)\right)-f\left(v^{0}(t, x)\right)= & f^{\prime}\left(v^{0}(t, x)+\xi^{\varepsilon}(t, x)\left(v^{\varepsilon}(t, x)-v^{0}(t, x)\right)\right) \\
& \times\left(v^{\varepsilon}(t, x)-v^{0}(t, x)\right) .
\end{aligned}
$$

Note that $f^{\prime}$ is Lipschitz continuous and $\xi^{\varepsilon}(t, x) \in(0,1)$; one can get

$$
\begin{array}{r}
\left|f^{\prime}\left(v^{0}(t, x)+\xi^{\varepsilon}(t, x)\left(v^{\varepsilon}(t, x)-v^{0}(t, x)\right)\right) \times\left(v^{\varepsilon}(t, x)-v^{0}(t, x)\right)\right| \\
\leq m^{\prime} \xi^{\varepsilon}(t, x)\left|v^{\varepsilon}(t, x)-v^{0}(t, x)\right| \leq m^{\prime}\left|v^{\varepsilon}(t, x)-v^{0}(t, x)\right| .
\end{array}
$$

So

$$
\begin{aligned}
\mathrm{III}^{\varepsilon}(t, x) & \leq m^{\prime} \int_{0}^{t} \int_{D} G_{\alpha}^{\delta}(t-s, x-z)\left|\left(v^{\varepsilon}(t, x)-v^{0}(t, x)\right) U^{\varepsilon}(s, z)\right| d s d z \\
& =\sqrt{\varepsilon} m^{\prime} \int_{0}^{t} \int_{D} G_{\alpha}^{\delta}(t-s, x-z)\left|U^{\varepsilon}(s, z)\right|^{2} d s d z
\end{aligned}
$$

Using Hölder's inequality, for $p<2$ and $1 / \alpha<q<\alpha$, one can get

$$
\begin{aligned}
E\left(\left|I I I^{\varepsilon}\right|_{\infty}^{t}\right)^{p} \leq & \varepsilon^{p / 2} m^{p}\left(\sup _{(t, x) \in[0, T] \times D}\left|\int_{0}^{t} \int_{D}\left(G_{\alpha}^{\delta}(t-s, x-z)\right)^{q} d s d z\right|\right)^{p / q} \\
& \times \int_{0}^{t} E\left(\left|U^{\varepsilon}\right|_{\infty}^{s}\right)^{2 p} d s,
\end{aligned}
$$

where $(1 / p)+(1 / q)=1$. Together with (5) of Lemma A.1 and Proposition 1, there exists a constant $C\left(p, m, m^{\prime}, T\right)$ only depending on $p, m, m^{\prime}, T$ satisfying

$$
E\left(\left|I I^{\varepsilon}\right|_{\infty}^{t}\right)^{p} \leq \varepsilon^{p / 2} C\left(p, M, M^{\prime}, T\right) .
$$

Since $\left|f^{\prime}\right| \leq M$, for $p>2$, together with Hölder's inequality and (5) of Lemma A.1, we can get

$$
\begin{aligned}
E\left(\left|\mathrm{IV}^{\varepsilon}\right|_{\infty}^{t}\right)^{p} \leq & M^{p}\left(\sup _{(t, x) \in[0, T] \times D}\left|\int_{0}^{t} \int_{D}\left(G_{\alpha}^{\delta}(t-s, x-z)\right)^{q} d s d z\right|\right)^{p / q} \\
& \times E \int_{0}^{t}\left(\left|U^{\varepsilon}-U\right|_{\infty}^{s}\right)^{p} d s,
\end{aligned}
$$

where $(1 / p)+(1 / q)=1$. Together with (52), (56), and (57), we can get

$$
E\left(\left|U^{\varepsilon}-U\right|_{\infty}^{t}\right)^{p} \leq C\left(p, m, m^{\prime}, T\right)\left(\varepsilon^{p / 2}+E \int_{0}^{t}\left(\left|U^{\varepsilon}-U\right|_{\infty}^{s}\right)^{p} d s\right) .
$$

Using Gronwall's inequality, one can get

$$
E\left(\left|U^{\varepsilon}-U\right|_{\infty}^{t}\right)^{p} \leq \varepsilon^{p / 2} C\left(p, m, m^{\prime}, T\right) \rightarrow 0, \quad \varepsilon \rightarrow 0,
$$

which implies Assumption 3 in Lemma 4. 
Now we prove Assumption 4 in Lemma 4. We will prove $\mathrm{III}^{\varepsilon}$ and $\mathrm{IV}^{\varepsilon}$ satisfy Assumption 4 in Lemma 4 . Using Hölder's inequality and (A.9), one can get

$$
\begin{aligned}
E\left|\mathrm{III}^{\varepsilon}(t, x)-\mathrm{III}^{\varepsilon}\left(t, x^{\prime}\right)\right|^{p} & \leq\left.\left. E\left|\sqrt{\varepsilon} m^{\prime} \int_{0}^{t} \int_{D}\left[G_{\alpha}^{\delta}(t-s, x-z)-G_{\alpha}^{\delta}\left(t-s, x^{\prime}-z\right)\right]\right| U^{\varepsilon}(s, z)\right|^{2} d s d z\right|^{p} \\
& \leq \varepsilon^{p / 2} M^{\prime p}\left(\left|\int_{0}^{t} \int_{D}\right| G_{\alpha}^{\delta}(t-s, x-z)-\left.G_{\alpha}^{\delta}\left(t-s, x^{\prime}-z\right)\right|^{q} d s d z\right)^{p / q} \times\left(\int_{0}^{t} \int_{D} E\left(\left|Y^{\varepsilon}(s, z)\right|_{\infty}^{s}\right)^{2 p} d s d z\right)^{1 / p} \\
& \leq \varepsilon^{p / 2} C\left(p, m, m^{\prime}, T\right)\left|x-x^{\prime}\right|^{p \theta^{\prime}}
\end{aligned}
$$

where $\theta^{\prime} \in(0, \min \{1,(\alpha+1) / q-1\})$ and $1 / p+1 / q=1$.

Similarly,

$$
\begin{aligned}
E\left|\mathrm{IV}^{\varepsilon}(t, x)-\mathrm{IV}^{\varepsilon}\left(t^{\prime}, x\right)\right|^{p} \leq & \left.\left.E\left|\sqrt{\varepsilon} m^{\prime} \int_{0}^{t} \int_{D}\left[G_{\alpha}^{\delta}(t-s, x-z)-G_{\alpha}^{\delta}\left(t^{\prime}-s, x-z\right)\right]\right| U^{\varepsilon}(s, z)\right|^{2} d s d z\right|^{p} \\
\leq & \left.\left.\varepsilon^{p / 2} C(p, m, T)\left|\int_{0}^{t} \int_{D}\left[G_{\alpha}^{\delta}(t-s, x-z)-G_{\alpha}^{\delta}\left(t^{\prime}-s, x-z\right)\right]\right| U^{\varepsilon}(s, z)\right|^{2} d s d z\right|^{p} \\
\leq & \left.\left.\varepsilon^{p / 2} C(p, m, T)\left|\int_{t^{\prime}}^{t} \int_{D}\left[G_{\alpha}^{\delta}(t-s, x-z)-G_{\alpha}^{\delta}\left(t^{\prime}-s, x-z\right)\right]\right| U^{\varepsilon}(s, z)\right|^{2} d s d z\right|^{p} \\
& +\left.\varepsilon^{p / 2} C(p, m, T)\left|\int_{0}^{t^{\prime}} \int_{D} G_{\alpha}^{\delta}(t-s, x-z) U^{\varepsilon}(s, z)\right|^{2} d s d z\right|^{p} \leq C\left(p, m, m^{\prime}, T\right)\left|t-t^{\prime}\right|^{p \mu},
\end{aligned}
$$

where $\mu^{\prime} \in(0,(\alpha+1 / q-1) / \alpha)$. Together with (89) and (90), we can get

$$
E \mid \operatorname{III}^{\varepsilon}(t, x)-\operatorname{III}^{\varepsilon}\left(\left.(s, z)\right|^{p} \leq C\left(|t-s|+|x-z|^{2}\right)^{\beta}\right.
$$

Similarly, one can get

$$
E \mid \operatorname{IV}^{\varepsilon}(t, x)-\operatorname{IV}^{\varepsilon}\left(\left.(s, z)\right|^{p} \leq C\left(|t-s|+|x-z|^{2}\right)^{\beta} .\right.
$$

Together with (91) and (92), we can get

$$
\begin{gathered}
E\left|\left(U^{\varepsilon}(t, x)-U(t, x)\right)-\left(U^{\varepsilon}(s, z)-U(s, z)\right)\right|^{p} \\
\leq C\left(|t-s|+|x-z|^{2}\right)^{\beta} .
\end{gathered}
$$

Then, for any $p>2$ and $q \in(1, \alpha)$ such that $\gamma \in(0, \beta / p)$ and $r \in[1, p)$, using Lemma 4 ,

$$
\lim _{\varepsilon \rightarrow 0} E\left(\left\|V^{\varepsilon}\right\|_{\gamma}\right)^{r}=0 .
$$

The proof of Theorem 2 is completed.
Proof of Theorem 3. By Theorem 1, U/a $(\varepsilon)$ obeys large deviation principles on $\mathscr{C}^{\gamma}([0, T] \times D)$, with the rate function $I$ given by (30) and the speed function $h^{2}(\varepsilon)$. Using Dembo and Zeitouni ([30] Theorem 4.2.13), to prove the large deviation principles of $U^{\varepsilon} / a(\varepsilon)$ is $e^{2}(\varepsilon)$-exponentially equivalent to $U / a(\varepsilon)$, that is,

$$
\limsup _{\varepsilon \rightarrow 0} e^{-2}(\varepsilon) \log P\left(\frac{\left\|U^{\varepsilon}-U\right\|_{\mu^{\prime}, \theta^{\prime}}}{a(\varepsilon)}>\delta\right)=-\infty
$$

holds for any $\delta>0$.

Since

$$
\left\|U^{\varepsilon}-U\right\|_{\gamma} \leq C\left(T, \mu^{\prime}, \theta^{\prime}\right)\left|U^{\varepsilon}-U\right|_{T, \gamma} .
$$

To prove (67), we only need to prove

$$
\limsup _{\varepsilon \rightarrow 0} e^{-2}(\varepsilon) \log P\left(\frac{\left|U^{\varepsilon}-U\right| t, \mu^{\prime}, \theta^{\prime}}{a(\varepsilon)}>\delta\right)=-\infty .
$$

Note the decomposition

$$
U^{\varepsilon}(t, x)-U(t, x)=\operatorname{III}^{\varepsilon}(t, x)+\operatorname{IV}^{\varepsilon}(t, x) .
$$


For any $q \in(1, \alpha), x, x^{\prime} \in[0,1],(1 / p)+(1 / q)=1$, and $0 \leq s \leq t \leq T$, by Hölder's inequality, (32), and (A.9), we can get

$$
\begin{aligned}
\left|\operatorname{IV}^{\varepsilon}(t, x)-\mathrm{IV}^{\varepsilon}\left(t, x^{\prime}\right)\right| & \leq m^{\prime}\left|\int_{0}^{t} \int_{D}\left[G_{\alpha}^{\delta}(t-s, x-z)-G_{\alpha}^{\delta}\left(t-s, x^{\prime}-z\right)\right]\left(U^{\varepsilon}(s, z)-U(s, z)\right) d s d z\right| \\
& \leq m^{\prime}\left(\left|\int_{0}^{t} \int_{D}\right| G_{\alpha}^{\delta}(t-s, x-z)-\left.G_{\alpha}^{\delta}\left(t-s, x^{\prime}-z\right)\right|^{q} d s d z\right)^{1 / q} \times\left(\int_{0}^{t} \int_{D}\left|U^{\varepsilon}(s, z)-U(s, z)\right|^{p} d s d z\right)^{1 / p} \\
& \leq M^{\prime}\left|x-x^{\prime}\right|^{\theta} \times\left(\int_{0}^{t}\left(\left|U^{\varepsilon}-U\right|_{\infty}^{u}\right)^{p}\right)^{1 / p} .
\end{aligned}
$$

where $\theta^{\prime} \in[0, \min \{1,(\alpha+1) / q-1\})$. Similarly,

$$
\begin{aligned}
\left|\mathrm{IV}^{\varepsilon}(t, x)-\mathrm{IV}^{\varepsilon}\left(t^{\prime}, x\right)\right| \leq & m^{\prime}\left|\int_{0}^{t^{\prime}} \int_{D}\left[G_{\alpha}^{\delta}(t-s, x-z)-G_{\alpha}^{\delta}\left(t^{\prime}-s, x-z\right)\right]\left(U^{\varepsilon}(s, z)-U(s, z)\right) d s d z\right| \\
\leq & m^{\prime}\left(\left|\int_{0}^{t} \int_{D}\right| G_{\alpha}^{\delta}(t-s, x-z)-\left.G_{\alpha}^{\delta}\left(t^{\prime}-s, x-z\right)\right|^{q} d s d z\right)^{1 / q} \times\left(\int_{0}^{t^{\prime}} \int_{D}\left|U^{\varepsilon}(s, z)-U(s, z)\right|^{p} d s d z\right)^{1 / p} \\
& +m^{\prime}\left(\left|\int_{t^{\prime}}^{t} \int_{D}\right| G_{\alpha}^{\delta}|(t-s, x-z)|^{q} d s d z\right)^{1 / q} \times\left(\int_{t^{\prime}}^{t} \int_{D}\left|U^{\varepsilon}(s, z)-U(s, z)\right|^{p} d s d z\right)^{1 / p} \\
\leq & m^{\prime}\left|t-t^{\prime}\right| \mu^{\prime} \times\left(\int_{0}^{t}\left(\left|U^{\varepsilon}-U\right|_{\infty}^{u}\right)^{p}\right)^{1 / p} .
\end{aligned}
$$

where $\mu^{\prime} \in(0,(\alpha+1) / q-1 / \alpha)$. Together with (99) and (100), we can get

$$
E \mid \mathrm{IV}^{\varepsilon}(\mathrm{t}, \mathrm{x})-\mathrm{IV}^{\varepsilon}\left(\left.(\mathrm{s}, \mathrm{z})\right|^{\mathrm{p}} \leq \mathrm{C}\left(|\mathrm{t}-\mathrm{s}|^{\mu \prime}+|\mathrm{x}-\mathrm{z}|^{\theta \prime}\right) .\right.
$$

Note that $\left|U^{\varepsilon}-U\right|_{s, \infty} \leq C\left(s, \mu^{\prime}, \theta^{\prime}\right)\left|U^{\varepsilon}-U\right|_{s, \gamma}$, we have

$$
\left|\mathrm{IV}^{\varepsilon}(t, x)\right|_{t, \gamma} \leq C\left(\int\left(C\left(s, \mu^{\prime}, \theta^{\prime}\right)\left|U^{\varepsilon}-U\right|_{s, \gamma}\right)^{p} d s\right) .
$$

Thus, for $t \in[0, T]$, we have

$$
\left(\left|U^{\varepsilon}-U\right|_{t, \gamma}\right)^{p} \leq C(p, m, \mathrm{~T})\left[\left(|\mathrm{III}|_{t, \gamma}\right)+\int_{0}^{t}\left(\left|\mathrm{U}^{\varepsilon}-\mathrm{U}\right|_{s, \gamma}\right)^{p} d s\right] .
$$

Applying Gronwall's Lemma to $f(t)=\left(\left|U^{\varepsilon}-U\right|_{t, \gamma}\right)^{p}$, we have

$$
\left(\left|U^{\varepsilon}-U\right|_{T, \gamma}\right)^{p} \leq C(p, T, m)\left(\left|\mathrm{III}^{\varepsilon}\right|_{T, \gamma}\right)^{p} .
$$

By (64) to prove (67), we only need to prove that for any $\delta>0$,

$$
\limsup _{\varepsilon \rightarrow 0} e^{-2}(\varepsilon) \log P\left(\frac{\left.\left.\right|^{\varepsilon}\right|_{\mathrm{T}, \gamma}}{a(\varepsilon)} \geq \delta\right)=-\infty .
$$

Note that

$$
\mathrm{III}^{\varepsilon}(t, x)=\int_{0}^{t} \int_{D} G_{\alpha}^{\delta}(t-s, \mathrm{x}-\mathrm{z}) \mathfrak{A}^{\varepsilon}(s, z) d s d z,
$$

where

$$
\mathfrak{A}^{\varepsilon}(s, z)=\left(\frac{f\left(v^{\varepsilon}(s, z)\right)-f\left(v^{0}(s, z)\right)}{\sqrt{\varepsilon}}-f^{\prime}\left(v^{0}(s, z)\right) U^{\varepsilon}(s, z)\right) .
$$

By the same method in the proof of (59), we have

$$
\left\|\mathrm{III}^{\varepsilon}\right\|_{T, \gamma} \leq \mathrm{C}\left(\mathrm{T}, \mu^{\prime}, \theta^{\prime}\right)\left|\mathfrak{A}^{\varepsilon}\right|_{T, \infty} .
$$


Similar to the proof of (53), we have

$$
\left|\mathfrak{\mathcal { I }}^{\varepsilon}\right|_{T, \infty} \leq m^{\prime} \frac{\left(\left|v^{\varepsilon}-v^{0}\right|_{T, \infty}\right)^{2}}{\sqrt{\varepsilon}}
$$

Together with (40) and (A.11), for any $t \in[0, T]$, we can get that

$$
\begin{aligned}
& \left|v^{\varepsilon}-v^{0}\right|_{t, \infty} \\
& \leq \sup _{(t, x) \in[0, T] \times D}\left|\int_{0}^{l} \int_{D} G_{\alpha}^{\delta}(l-s, x-z) m\right| v^{\varepsilon}(s, z)-v^{0}(s, z)|d s d z| \\
& \cdot \sup _{(t, x) \in[0, T] \times D}\left|\sqrt{\varepsilon} \int_{0}^{l} \int_{D} G_{\alpha}^{\delta}(t-s, x-z) B^{H}(d s d z)\right| \\
& \leq C(m, T) \int_{0}^{t}\left|v^{\varepsilon}-v^{0}\right|_{s, \infty} d s+\left|\operatorname{IIV}^{\varepsilon}\right|_{t, \infty},
\end{aligned}
$$

where

$$
\begin{aligned}
\operatorname{IIV}^{\varepsilon}(t, x) & =\sqrt{\varepsilon} \int_{0}^{1} \int_{D} G_{\alpha}^{\delta}(t-s, x-z) B^{H}(d s d z) \\
& =\sqrt{\varepsilon} \int_{0}^{1} \int_{D} K_{H}^{*} G_{\alpha}^{\delta}(t-s, x-z) W(d s d z) .
\end{aligned}
$$

By Gronwall's Lemma, one can get

$$
\left|v^{\varepsilon}-v^{0}\right|_{T, \infty} \leq C(M, T)\left|\operatorname{IIV}^{\varepsilon}\right|_{T, \infty} \leq C(M, T)\left|\operatorname{IIV}^{\varepsilon}\right| \gamma
$$

Applying Lemma 4 with

$$
\begin{aligned}
& F(t, x, s, z)=K_{H}^{*} G(t-s, x-z) 1_{s \leq t}, \beta_{0}=\frac{1}{2}, C_{F}=C \\
& \left.\rho=\sqrt{\varepsilon} M\left(1+\left|v^{0}\right|_{T, \infty}+\theta\right), N(t, x)=\left.\sqrt{\varepsilon} 1_{\left[\left|v^{\varepsilon}\right|\right.}\right|_{T, \infty}<\left|v^{0}\right|_{T, \infty}+\theta\right]
\end{aligned}
$$

for any fixed $\theta>0$, one can get that for any $\beta, G \geq \sqrt{\varepsilon} K(1+$ $\left.\left.v^{0}\right|_{T, \infty}+\theta\right) C_{F}^{1 / 2} C\left(\beta, \beta_{0}\right) C\left(\beta, \beta_{0}\right)$,

$$
\begin{aligned}
& P\left(\left.\mathrm{IIV}^{\varepsilon}\right|_{\beta, \beta} \geq \mathrm{M},\left|\mathrm{v}^{\varepsilon}\right|_{T, \infty}<\left|\mathrm{v}^{0}\right|_{\mathrm{T}, \infty}+\theta\right) \\
& \quad \leq L \exp \left(-\frac{G^{2}}{\varepsilon M^{2} C C^{2}\left(\beta, \beta_{0}\left(1+\left.v^{0}\right|_{T, \infty}+\theta\right)^{2}\right)}\right)
\end{aligned}
$$

We can get

$$
\begin{aligned}
\limsup _{\varepsilon \rightarrow 0} e^{-2}(\varepsilon) \log P\left(\left.v^{\varepsilon}\right|_{T, \infty} \geq\left|v^{0}\right|_{T, \infty}+\theta\right) \\
\quad \leq \limsup _{\varepsilon \rightarrow 0} e^{-2}(\varepsilon) \log P\left(v^{\varepsilon}-\left.v^{0}\right|_{T, \infty} \geq \theta\right)=-\infty
\end{aligned}
$$

Together with (73), (78), (82), (83), and (85), we have

$$
\begin{aligned}
\limsup _{\varepsilon \rightarrow 0} & e^{-2}(\varepsilon) \log P\left(\left(\frac{\|\mathrm{III}\|_{\mathrm{T}, \mu^{\prime}, \theta^{\prime}}}{a(\varepsilon)}\right)>\delta\right) \\
& \leq \limsup _{\varepsilon \rightarrow 0} e^{-2}(\varepsilon) \log P\left(\left(\|\mathrm{III}\|_{\mathrm{T}, \mu^{\prime}, \theta^{\prime}}\right)^{2}>\frac{\sqrt{\varepsilon} \mathrm{a}(\varepsilon) \delta}{\mathrm{C}(\beta, \mathrm{T}, \mathrm{m})}\right) \\
& \leq \limsup _{\varepsilon \rightarrow 0} e^{-2}(\varepsilon) \log \left[P\left(\left(\|\mathrm{III}\| \mathrm{T}, \mu^{\prime}, \theta^{\prime}\right)^{2}>\frac{\sqrt{\varepsilon} \mathrm{a}(\varepsilon) \delta}{C(\beta, \mathrm{T}, \mathrm{m})},\left|\mathrm{v}^{\varepsilon}\right|_{\mathrm{T}, \infty}<\left|\mathrm{v}^{0}\right|_{\mathrm{T}, \infty}+\theta\right)+P\left(\left|v^{\varepsilon}\right|_{T, \infty} \geq\left|v^{0}\right|_{T, \infty}+\theta\right)\right] \\
& \leq\left(\limsup _{\varepsilon \rightarrow 0} \frac{-\delta}{\sqrt{\varepsilon} a(\varepsilon) C(\beta, T, m) m^{2} C C^{2}\left(\beta, \beta_{0}\right)\left(1+\left.v^{0}\right|_{T, \infty}+\theta\right)^{2}}\right) v\left(\limsup _{\varepsilon \rightarrow 0}^{-2}(\varepsilon) \log P\left(\left.v^{\varepsilon}\right|_{T, \infty} \geq\left|v^{0}\right|_{T, \infty}+\theta\right)\right)=-\infty
\end{aligned}
$$

This completes the proof.

\section{Appendix}

\section{Green Function}

The nonlocal factional differential operator $D_{\delta, \alpha}$ is defined by

$$
\mathscr{F}\left(D_{\delta, \alpha} \phi\right)(\xi)=-|\xi|^{\alpha} \exp \left(-i \delta \frac{\pi}{2} \operatorname{sgn}(\xi)\right) \mathscr{F}(\phi)(\xi),
$$

where $\alpha$ is called the index of stability and $\delta$ improperly referred to as the skewness which satisfy $|\delta| \leq \min \left\{\alpha-[\alpha]_{2}\right.$, $\left.2+[\alpha]_{2}-\alpha\right\}$ and $\delta=0$ when $\delta \in 2 \mathbb{N}+1$.

The operator $D_{\delta, \alpha}$ is a closed, densely defined operator on $L^{2}(\mathbb{R})$, and it is the infinitesimal generator of a semigroup which is in general not symmetric and not a contraction. This operator is a generalization of various well-known operators, such as the Laplacian operator (when $\alpha=2$ ), the inverse of the generalized Riesz-Feller potential (when $\alpha>2$ ), and the 
Riemann-Liouville differential operator (when $|\delta|=2+[\alpha]_{2}$ or $|\delta|=\alpha-[\alpha]$ ). It is self-adjoint only when $\delta=0$, and in this case, it coincides with the fractional power of the Laplacian. We refer the readers to Debbi and Dozzi [31] for more details about this operator.

According to Komatsu [32], $D_{\delta, \alpha}$ can be represented for $1<\alpha<2$, by

$$
\begin{aligned}
& D_{\delta, \alpha} \varphi(x) \\
& \quad=\int_{0}^{1} \frac{\varphi(x+y)-\varphi(x)-y \varphi^{\prime}(x)}{|y|^{1+\alpha}}\left(\kappa_{-}^{\delta} 1_{(-\infty, 0)}(y)+\kappa_{+}^{\delta} 1_{(-0,+\infty)}(y)\right) d y,
\end{aligned}
$$

and for $0<\alpha<1$, by

$D_{\delta, \alpha} \varphi(x)=\int_{0}^{1} \frac{\varphi(x+y)-\varphi(x)}{|y|^{1+\alpha}}\left(\kappa_{-}^{\delta} 1_{(-\infty, 0)}(y)+\kappa_{+}^{\delta} 1_{(-0,+\infty)}(y)\right) d y$,

where $\kappa_{-}^{\delta}$ are $\kappa_{+}^{\delta}$ two nonnegative constants satisfying $\kappa_{-}^{\delta}+$ $\kappa_{+}^{\delta}>0, \varphi$ is a smooth function for which the integral exists, and $\varphi^{\prime}$ is its derivative. This representation identifies it as the infinitesimal generator for a nonsymmetric $\alpha$-stable Lévy process.

Suppose $G_{\alpha}^{\delta}(t, x)$ is the fundamental solution to the following equation:

$$
\begin{aligned}
\frac{\partial v}{\partial t}(t, x) & =D_{\delta, \alpha}(t, x), \\
v(0, x) & =\delta_{0}(x), \quad t>0, x \in \mathbb{R},
\end{aligned}
$$

where $\delta_{0}(\cdot)$ is the Dirac distribution. Using Fourier transform, one can get $G_{\alpha}^{\delta}(t, x)$ which is given by

$$
G_{\alpha}^{\delta}(t, x)=\frac{1}{2 \pi} \int_{D} \exp \left(-i z x-t|z|^{\alpha} \exp \left(-i \delta \frac{\pi}{2} \operatorname{sgn}(z)\right)\right) d z .
$$

Let us list some known facts on $G_{\alpha}^{\delta}(t, x)$ which will be used later on (see, e.g., Debbi and Dozzi [31]).

Lemma A.1. Suppose $\alpha \in(0, \infty) /\{\mathbb{N}\}$, one can get the following:

(1) $G_{\alpha}^{\delta}(t, \cdot)$ is not symmetric with respect to $x$.

(2) For any $x \in \mathbb{R}$ and $s, t \in(0, \infty)$,

$$
\frac{\partial^{n}}{\partial x^{n}} G_{\alpha}^{\delta}(t, x)=(s)^{-((n+t) / \alpha)} G_{\alpha}^{\delta}\left(s^{-1} t, s^{-(1 / \alpha)} x\right)
$$

or equivalently

$$
\frac{\partial^{n}}{\partial x^{n}} G_{\alpha}^{\delta}(t, x)=(t)^{-((n+t) / \alpha)} G_{\alpha}^{\delta}\left(1,(t)^{-(1 / \alpha)} x\right) .
$$

(3) For any $s, t \in(0, \infty), G_{\alpha}^{\delta}(s, \cdot) * G_{\alpha}^{\delta}(t, \cdot)=G_{\alpha}^{\delta}(s+t, \cdot)$

(4) For $n \geq 1$, there exist constants $C$ and $C_{n}>0$ satisfying

$$
\left|G_{\alpha}^{\delta}(1, x)\right| \leq C \frac{1}{1+|x|^{1+\alpha}}, \quad\left|\frac{\partial^{n}}{\partial x^{n}} G_{\alpha}^{\delta}(1, x)\right| \leq C_{n} \frac{|x|^{\alpha+n-1}}{\left(1+|x|^{\alpha+n}\right)^{2}} .
$$

(5) $\int_{0}^{T} \int_{D}\left|G_{\alpha}^{\delta}(t, x)\right|^{\lambda} d t d x<\infty$ if and only if $(1 / \alpha)<\lambda<\alpha$.

Lemma A.2. For $\theta \in[0, \min \{1,(\alpha+1) H-1\})$ and $\mu \in$ $[0,((\alpha+1) H-1) / \alpha)$, we have

$$
\left[\int_{0}^{t} \int_{\mathbb{R}}\left|G_{\alpha}^{\delta}(t-v, y-w)-G_{\alpha}^{\delta}(t-v, y-w)\right|^{1 / H} d w d v\right]^{H} \leq m|x-y|^{\theta},
$$

$$
\left[\int_{0}^{t} \int_{\mathbb{R}}\left|G_{\alpha}^{\delta}(t-v, x-w)-G_{\alpha}^{\delta}(s-v, x-w)\right|^{1 / H} d w d v\right]^{H} \leq m|t-s|^{\mu},
$$

$$
\left[\int_{s}^{t} \int_{\mathbb{R}}\left|G_{\alpha}^{\delta}(t-v, x-w)\right|^{1 / H} d w d v\right]^{H} \leq m|t-s|^{((\alpha+1) H-1) / \alpha},
$$

where $m>0$ is a constant.

Proof. For any $x, y \in \mathbb{R}$ and $t \in[0, T]$,

$$
\begin{gathered}
{\left[\int_{0}^{t} \int_{\mathbb{R}}\left|G_{\alpha}^{\delta}(t-v, x-w)-G_{\alpha}^{\delta}(t-v, y-w)\right|^{1 / H} d w d v\right]^{H}} \\
=\left\|G_{\alpha}^{\delta}(t-*, x-\cdot)-G_{\alpha}^{\delta}(t-*, y-\cdot)\right\|_{L^{1 / H}([0, T] \times \mathbb{R})} \\
=\|\left|G_{\alpha}^{\delta}(t-*, x-\cdot)-G_{\alpha}^{\delta}(t-*, y-\cdot)\right|^{\theta} \\
\cdot\left|G_{\alpha}^{\delta}(t-*, x-\cdot)-G_{\alpha}^{\delta}(t-*, y-\cdot)\right|^{1-\theta} \|_{L^{1 / H}([0, T] \times \mathbb{R})} \\
\leq \|\left|G_{\alpha}^{\delta}(t-*, x-\cdot)-G_{\alpha}^{\delta}(t-*, y-\cdot)\right|^{\theta} \\
\cdot\left|G_{\alpha}^{\delta}(t-*, x-\cdot)\right|^{1-\theta} \|_{L^{1 / H}([0, T] \times \mathbb{R})}=I_{1}+I_{2} . \\
+||\left|G_{\alpha}^{\delta}(t-*, x-\cdot)-G_{\alpha}^{\delta}(t-*, y-\cdot)\right|^{\theta} \\
\cdot\left|G_{\alpha}^{\delta}(t-*, y-\cdot)\right|^{1-\theta} \|_{L^{1 / H}([0, T] \times \mathbb{R})}
\end{gathered}
$$


By the mean-value theorem, for $\theta \in[0,1)$, one can get that

$$
\begin{aligned}
\mathrm{I}_{1} & =|||x-y|^{\theta}\left|\frac{\partial}{\partial x} G_{\alpha}^{\delta}(t-*, \eta-\cdot)\right|^{\theta} \cdot\left|G_{\alpha}^{\delta}(t-*, x-\cdot)\right|^{1-\theta} \|_{L^{1 / H}([0, T] \times \mathbb{R})} \\
& =\left(|x-y|^{\theta / H} \int_{0}^{\partial} \int_{\mathbb{R}}\left|\frac{\partial}{\partial x} G_{\alpha}^{\delta}(t-u, \eta-w)\right|^{\theta / H}\left|G_{\alpha}^{\delta}(t-v, x-w)\right|^{(1-\theta) / H} d w d u\right)^{H}
\end{aligned}
$$

and

$$
\begin{aligned}
\int_{\mathbb{R}}\left|\frac{\partial}{\partial x} G_{\alpha}^{\delta}(t-u, \eta-w)\right|^{\theta / H}\left|G_{\alpha}^{\delta}(t-u, x-w)\right|^{(1-\theta) / H} d w \\
\quad=(t-u)^{(1 / \alpha)-((1+\theta) / \alpha H)} \int_{\mathbb{R}}\left|\frac{\partial}{\partial x} G_{\alpha}^{\delta}(1, \tilde{\eta}-w)\right|^{\theta / H}\left|G_{\alpha}^{\delta}(1, w)\right|^{(1-\theta) / H} d w \\
\quad \leq C_{H, \theta}(t-u)^{(1 / \alpha)-((1+\theta) / \alpha H)} .
\end{aligned}
$$

Therefore, if $(1 / \alpha)-((1+\theta) / \alpha H)+1>0$, that is, $\theta<$ $(\alpha+1) H-1$, then,

$$
I_{1} \leq C_{T, \theta, H}|x-w|^{\theta} .
$$

Similarly, we can check

$$
I_{2} \leq C_{T, \theta, H}|x-w|^{\theta} .
$$

$$
\begin{gathered}
{\left[\int_{0}^{t} \int_{\mathbb{R}}\left|G_{\alpha}^{\delta}(t-v, x-w)-G_{\alpha}^{\delta}(s-v, x-w)\right|^{1 / H} d z d v\right]^{H}} \\
=\left\|\mid G_{\alpha}^{\delta}(t-*, x-\cdot)-G_{\alpha}^{\delta}(s-*, x-\cdot)\right\|_{L^{1 / H}([0, T] \times \mathbb{R})} \\
=\||| G_{\alpha}^{\delta}(t-*, x-\cdot)-\left.G_{\alpha}^{\delta}(s-*, x-\cdot)\right|^{\mu} \\
\cdot|| G_{\alpha}^{\delta}(t-*, x-\cdot)-\left.G_{\alpha}^{\delta}(s-*, x-\cdot)\right|^{1-\mu} \|_{L^{1 / H}([0, T] \times \mathbb{R})} \\
\leq \|\left|G_{\alpha}^{\delta}(t-*, x-\cdot)-G_{\alpha}^{\delta}(s-*, x-\cdot)\right|^{\mu} \\
\cdot\left|G_{\alpha}^{\delta}(s-*, x-\cdot)\right|^{1-\mu} \|_{L^{1 / H}([0, T] \times \mathbb{R})}:=\mathrm{II}_{1}+\mathrm{II}_{2} .
\end{gathered}
$$

Hence, the inequality (A.9) holds. As for the inequality (A.10), for any $x \in \mathbb{R}$ and $t, s \in[0, T]$,
By mean-value theorem, it holds that

$$
\begin{aligned}
\mathrm{II}_{1} & =|t-s|^{\mu}\left|\frac{\partial}{\partial t} G_{\alpha}^{\delta}(\rho-*, x-\cdot)\right|^{\mu} \cdot\left|G_{\alpha}^{\delta}(t-*, x-\cdot)\right|^{1-\mu} \|_{L^{1 / H}([0, T] \times \mathbb{R})} \\
& =\left(\int_{0}^{T} \int_{\mathbb{R}}|t-s|^{\mu / H}\left|\frac{\partial}{\partial t} G_{\alpha}^{\delta}(\rho-r, x-w)\right|^{\mu / H}\left|G_{\alpha}^{\delta}(t-r, x-w)\right|^{(1-\mu) / H} d w d u\right)^{H} .
\end{aligned}
$$

Note that

$$
G_{\alpha}^{\delta}(t, y)=t^{-1 / \alpha} G_{\alpha}^{\delta}\left(1, t^{-(1 / \alpha)} y\right)
$$

$$
\begin{aligned}
\frac{\partial}{\partial t} G_{\alpha}^{\delta}(t, y)= & \left(-\frac{1}{\alpha}\right) t^{-(1-(1 / \alpha))} G_{\alpha}^{\delta}\left(1, t^{-(1 / \alpha)} y\right) \\
& +\left(-\frac{1}{\alpha}\right) t^{-(1-2(1 / \alpha))}\left[\left.y \frac{\partial}{\partial z} G_{\alpha}^{\delta}(1, w)\right|_{w=t^{-(1 / \alpha)} y}\right]
\end{aligned}
$$




$$
\begin{aligned}
& \int_{\mathbb{R}}\left|\frac{\partial}{\partial t} G_{\alpha}^{\delta}(\rho-r, x-w)\right|^{\mu / H}\left|G_{\alpha}^{\delta}(t-r, x-w)\right|^{(1-\mu) / H} d w \\
& \left.\leq C_{\alpha, H} \int_{\mathbb{R}}(t-r)^{(-1-(1 / \alpha))(\mu / H)-(1 / \alpha)((1-u) / H)}\left|G_{\alpha}^{\delta}\left(1,(\rho-u)^{-(1 / \alpha)}(x-w)\right)\right|^{\mu / H} \cdot \mid G_{\alpha}^{\delta}\left(1,(t-u)^{-(1 / \alpha)}(x-w)\right)\right)\left.\right|^{(1-\mu) / H} d w \\
& +C_{\alpha, H} \int_{\mathbb{R}}(t-r)^{(-1-(2 / \alpha))(\mu / H)-(1 / \alpha)((1-u) / H)}\left[\left.|x-w|^{\mu / H}\left|\frac{\partial}{\partial w} G_{\alpha}^{\delta}(1, w)\right|_{w=(\rho-r))^{-(1 / \alpha)}(x-w)}\right|^{\mu / H} \cdot\left|G_{\alpha}^{\delta}\left(1,(t-u)^{-(1 / \alpha)}(x-w)\right)\right|^{(1-\mu) / H} d w\right] \\
& \leq C_{\alpha, H}(t-u)^{-\left((\alpha \mu+1) / H_{\alpha}\right)+(1 / \alpha)}+C_{\alpha, H}(t-u)^{-\left((\alpha \mu+\mu+1) / H_{\alpha}\right)+(1 / \alpha)} \text {. }
\end{aligned}
$$

Therefore, if $-((\alpha \mu+1) / H \alpha)+(1 / \alpha)+(1>0)$ i.e. $\quad \mu<$ $((\alpha+1) H-1) / \alpha$, then, for $\mu \in[0,((a+1) H-1) / \alpha)$,

$$
\mathrm{II}_{1} \leq \mathrm{C}_{\alpha, \mathrm{H}, \mathrm{T}, \mu}|t-s|^{\mu} .
$$

Similarly,

$$
\mathrm{II}_{2} \leq \mathrm{C}_{\alpha, \mathrm{H}, \mathrm{T}, \mu}|\mathrm{t}-\mathrm{s}|^{\mu} .
$$

So (A.10) holds. Now let us prove that (A.11) holds. For any $x \in \mathbb{R}$ and $s, t \in[0, T]$,

$$
\begin{aligned}
& {\left[\int_{s}^{t} \int_{\mathbb{R}}\left|G_{\alpha}^{\delta}(t-v, x-w)\right|^{1 / H} d w d u\right]^{H}} \\
& \quad \leq\left[\int_{s}^{t}((t-u))^{-(1 / \alpha H)+(1 / \alpha)}\left(\int_{\mathbb{R}}\left|G_{\alpha}^{\delta}(1, z)\right|^{1 / H} d w\right) d v\right]^{H} \\
& \quad \leq C_{H}(t-s)^{((a+1) H-1) / \alpha}
\end{aligned}
$$

The proof of the lemma is completed.

Lemma A.3. Suppose $p \in[1, \infty), q \in[1, p]$, and $\rho \in[1, \infty)$ satisfying

$$
\frac{1}{\rho}=\frac{1}{p}-\frac{1}{q}+1 \in[0,1]
$$

Suppose $G_{\alpha}^{\delta}=G_{\alpha}^{\delta}(t, x-z)$ is the Green kernel, $Q=(\partial / \partial z)$ $G_{\alpha}^{\delta}$ or $Q=G_{\alpha}^{\delta}$ with $(t, x, z) \in[0, T] \times \mathbb{R} \times \mathbb{R}$. We define I by

$$
I(u)(t, x)=\int_{0}^{t} \int_{\mathbb{R}} Q(t-s, x-z) u(s, z) d s d s
$$

with $\quad u \in L^{1}\left([0, T] ; L^{q}()\right) . \quad$ Then, $\quad I: L^{1}\left([0, T] ; L^{q}()\right) \rightarrow$ $L^{\infty}\left([0, T] ; L^{q}()\right)$ is a bounded linear operator which satisfies the following:
(1) When $Q=(\partial / \partial y) G_{\alpha}^{\delta}$, then, for all $r \in[1,(1+\alpha / 2))$,

$$
\begin{aligned}
& \|I(u)(t, \cdot)\|_{p} \\
& \quad \leq C \int_{0}^{t}(t-s)^{-(2 / \alpha)+(1 / \alpha r)}\|u(x, \cdot)\|_{q} d s, \quad \forall t \in[0, T] .
\end{aligned}
$$

(2) When $Q=G_{\alpha}^{\delta}$, then, for all $\rho \in[1,1+\alpha)$,

$$
\begin{aligned}
& \|I(u)(t, \cdot)\|_{p} \\
& \quad \leq C \int_{0}^{t}(t-s)^{-(1 / \alpha)+(1 / \alpha r)}\|u(x, \cdot)\|_{q} d s, \quad \forall t \in[0, T] .
\end{aligned}
$$

Proof. Together with Minkowski's inequality, Young inequality and (4) of Lemma A.1. We can get

$$
\begin{aligned}
\|I(u)(t, \cdot)\|_{p}= & \left\|\int_{0}^{t} \int_{\mathbb{R}} \frac{\partial}{\partial z} G_{\alpha}^{\delta}(t-s, \cdot-z) v(s, z) d z d s\right\|_{p} \\
\leq & \int_{0}^{t}\left\|\int_{\mathbb{R}} \frac{\partial}{\partial z} G_{\alpha}^{\delta}(t-s, \cdot-z) u(s, z) d z\right\|_{p} d s \\
\leq & C \int_{0}^{t}(t-s)^{-(2 / a)} \\
& \cdot\left\|\int_{\mathbb{R}} \frac{\partial}{\partial z} G_{\alpha}^{\delta}\left(1,(t-s)^{-(1 / a)}(\cdot-z)\right)|u(s, z)| d z\right\|_{p} d s \\
\leq & C \int_{0}^{t}(t-s)^{-(2 / a)} \\
& \cdot\left\|\left(\frac{\partial}{\partial z} G_{\alpha}^{\delta}\left(1,(t-s)^{-(1 / a)} \cdot\right) *|u(s, \cdot)|\right)(\cdot)\right\|_{p} d s \\
\leq & C \int_{0}^{t}(t-s)^{-(2 / a)}\left\|\frac{\partial}{\partial y} G_{\alpha}^{\delta}\left(1,(t-s)^{-(1 / a)} \cdot\right)\right\|_{p} \\
& \cdot\|u(s, \cdot)\|_{q} d s \\
\leq & \int_{0}^{t}(t-s)^{-(2 / a)+(1 / \alpha \rho)}\|u(s, \cdot)\|_{q} d s,
\end{aligned}
$$


where one has used the result that, for $\rho \in[1,(1+\alpha) / 2)$,

$$
\begin{aligned}
\left.\| G_{\alpha}^{\delta}\left(1,(t-s)^{-(1 / \alpha)}\right)\right) \|_{\rho} & =\left[\int_{\mathbb{R}} \frac{\partial}{\partial z}\left(G_{\alpha}^{\delta}\left(1,(t-s)^{-(1 / \alpha)} z\right)\right)^{\rho} d z\right]^{1 / p} \\
& \leq(t-s)^{1 / \alpha \rho}\left[\int_{\mathbb{R}} \frac{\partial}{\partial y}\left(G_{\alpha}^{\delta}(1, y)\right)^{\rho} d y\right]^{1 / p} \\
& \leq C(t-s)^{1 / \alpha \rho} .
\end{aligned}
$$

The proof of (2) is omitted since it is similar to case (1). The proof of this lemma is completed.

\section{Data Availability}

No data were used to support this study.

\section{Conflicts of Interest}

The authors declare that there is no conflict of interest regarding the publication of this article.

\section{Acknowledgments}

Xichao Sun is partially supported by the Natural Science Foundation of Anhui Province (1808085MA02) and the Natural Science Foundation of Bengbu University (2017GJPY04, 2016KYTD02, 2017jxtd2, and 2017ZR08). Ming Li is partially supported by National Natural Science Foundation of China under the project Grant nos. 61672238, 61272402, and 61070214 .

\section{References}

[1] M. I. Freidlin and A. D. Wentzell, Random Perturbations of Dynamical Systems, Springer-Verlag, New York, NY, USA, 1984.

[2] C. Cardon-Weber, "Large deviations for a Burgers'-type SPDE," Stochastic Processes and their Applications, vol. 84, no. 1, pp. 53-70, 1999.

[3] D. Marquez-Carreras and M. Sarra, "Large deviation principle for a stochastic heat equation with spatially correlated noise," Electronic Journal of Probability, vol. 8, no. 12, 2003.

[4] T. El Mellali and M. Mellouk, "Large deviations for a fractional stochastic heat equation in spatial dimension $\mathbb{R}^{d}$ driven by a spatially correlated noise," Stochastics and Dynamics, vol. 16, no. 1, article 1650001, 2016.

[5] Y. M. Jiang, K. H. Shi, and Y. J. Wang, "Large deviation principle for the fourth-order stochastic heat equations with fractional noises," Acta Mathematica Sinica, English Series, vol. 26, no. 1, pp. 89-106, 2010.

[6] A. Budhiraja, P. Dupuis, and M. Fischer, "Large deviation properties of weakly interacting processes via weak convergence methods," The Annals of Probability, vol. 40, no. 1, pp. 74-102, 2012.

[7] A. Budhiraja, W. T. Fan, and R. Wu, "Large deviations for Brownian particle systems with killing," Journal of Theoretical Probability, vol. 6, pp. 1-40, 2016.
[8] W. Liming, "Moderate deviations of dependent random variables related to CLT," The Annals of Probability, vol. 23, no. 1, pp. 420-445, 1995.

[9] A. Guillin and R. Liptser, "Examples of moderate deviation principle for diffusion processes," Discrete and Continuous Dynamical Systems - Series B, vol. 6, no. 4, pp. 803-828, 2006.

[10] C. Cattani and A. Ciancio, "On the fractal distribution of primes and prime-indexed primes by the binary image analysis," Physica A: Statistical Mechanics and its Applications, vol. 460, pp. 222-229, 2016.

[11] A. Budhiraja, P. Dupuis, and A. Ganguly, "Moderate deviation principles for stochastic differential equations with jumps," The Annals of Probability, vol. 44, no. 3, pp. 1723-1775, 2016.

[12] R. Wang and T. Zhang, "Moderate deviations for stochastic reaction-diffusion equations with multiplicative noise," Potential Analysis, vol. 42, no. 1, pp. 99-113, 2015.

[13] Y. Li, R. Wang, and S. Zhang, "Moderate deviations for a stochastic heat equation with spatially correlated noise," Acta Applicandae Mathematicae, vol. 139, no. 1, pp. 59-80, 2015.

[14] J. Yang and Y. Jiang, "Moderate deviations for fourth-order stochastic heat equations with fractional noises," Stochastics and Dynamics, vol. 16, no. 6, article 1650022, 2016.

[15] J. Droniou and C. Imbert, "Fractal first-order partial differential equations," Archive for Rational Mechanics and Analysis, vol. 182, no. 2, pp. 299-331, 2006.

[16] E. G. Bakhoum and C. Toma, "Alternating coordinatemomentum representation for quantum states based on bopp operators for modelling long-distance coherence aspects," Mathematical Problems in Engineering, vol. 2015, Article ID 818019, 7 pages, 2015.

[17] C. Levy and M. Pinchas, "Maximum likelihood estimation of clock skew in IEEE 1588 with fractional Gaussian noise," Mathematical Problems in Engineering, vol. 2015, Article ID 174289, 24 pages, 2015.

[18] A. Mardani, M. R. Hooshmandasl, M. H. Heydari, and C. Cattani, "A meshless method for solving the time fractional advection-diffusion equation with variable coefficients," Computers \& Mathematics with Applications, vol. 75, no. 1, pp. 122-133, 2018.

[19] O. Niculescu, D. G. Dimitriu, V. P. Paun, P. D. Matasaru, D. Scurtu, and M. Agop, "Experimental and theoretical investigations of a plasma fireball dynamics," Physics of Plasmas, vol. 17, no. 4, article $042305,2010$.

[20] V. Paun, "Fractal surface analysis of Zircaloy-4 SEM micrographs using the time-series method," Central European Journal of Physics, vol. 7, no. 2, 2009.

[21] M. Pinchas, "Symbol error rate for nonblind adaptive equalizers applicable for the SIMO and FGn case," Mathematical Problems in Engineering, vol. 2014, Article ID 606843, 11 pages, 2014.

[22] C. Mueller, "The heat equation with Lévy noise," Stochastic Processes and their Applications, vol. 74, no. 1, pp. 67-82, 1998.

[23] J. Wu, "Fractal Burgers equation with stable Lévy noise," in International Conference SPDE and Applications-VII, pp. 4-10, 2004.

[24] J. Liu, L. Yan, and Y. Cang, "On a jump-type stochastic fractional partial differential equation with fractional noises," Nonlinear Analysis: Theory, Methods \& Applications, vol. 75, no. 16, pp. 6060-6070, 2012.

[25] D. Wu, "On the solution process for a stochastic fractional partial differential equation driven by space-time white noise," 
Statistics \& Probability Letters, vol. 81, no. 8, pp. 11611172, 2011.

[26] J. B. Walsh, "An introduction to stochastic partial differential equations," in École d'Été de Probabilités de Saint Flour $X I V$ - 1984, vol. 1180 of Lecture Notes in Mathematics, pp. 265-439, Springer, Berlin, Heidelberg, 1986.

[27] D. Nualart and Y. Ouknine, "Regularization of quasilinear heat equations by a fractional noise," Stochastics and Dynamics, vol. 4, no. 2, pp. 201-221, 2004.

[28] H. Doss and P. Priouret, "Petites perturbations de systemes dynamiques avec reflexion," in Séminaire de Probabilités XVII 1981/82, vol. 986 of Lecture Notes in Mathematics, pp. 353370, Springer, Berlin, Heidelberg, 1983.

[29] V. Bally, A. Millet, and M. Sanz-Sole, "Approximation and support theorem in Holder norm for parabolic stochastic partial differential equations," The Annals of Probability, vol. 23, no. 1, pp. 178-222, 1995.

[30] A. Dembo and O. Zeitouni, Large Deviations Techniques and Applications, Springer-Verlag, Second edition, 1998.

[31] L. Debbi and M. Dozzi, "On the solutions of nonlinear stochastic fractional partial differential equations in one spatial dimension," Stochastic Processes and their Applications, vol. 115, no. 11, pp. 1764-1781, 2005.

[32] T. Komatsu, "On the martingale problem for generators of stable processes with perturbations," Osaka Journal of Mathematics, vol. 21, pp. 113-132, 1984. 


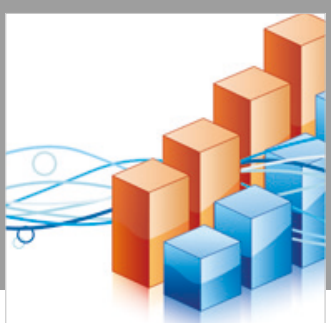

Advances in

Operations Research

\section{-n-m}
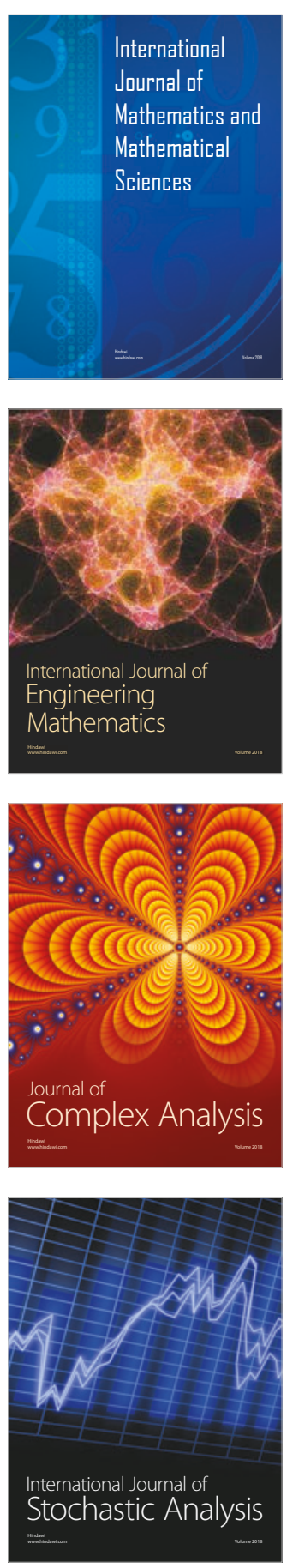
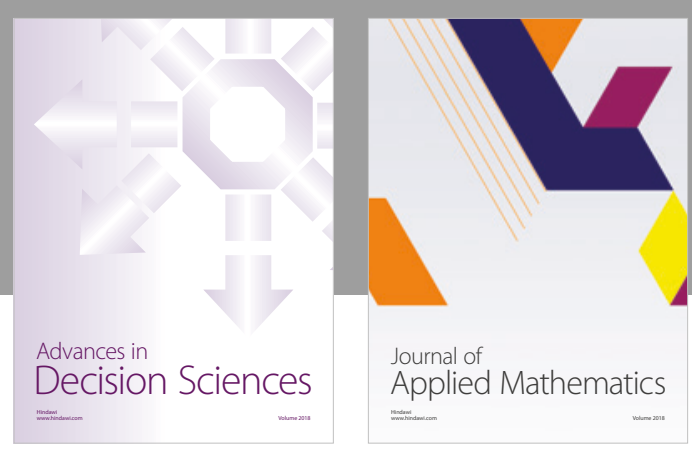

Journal of

Applied Mathematics
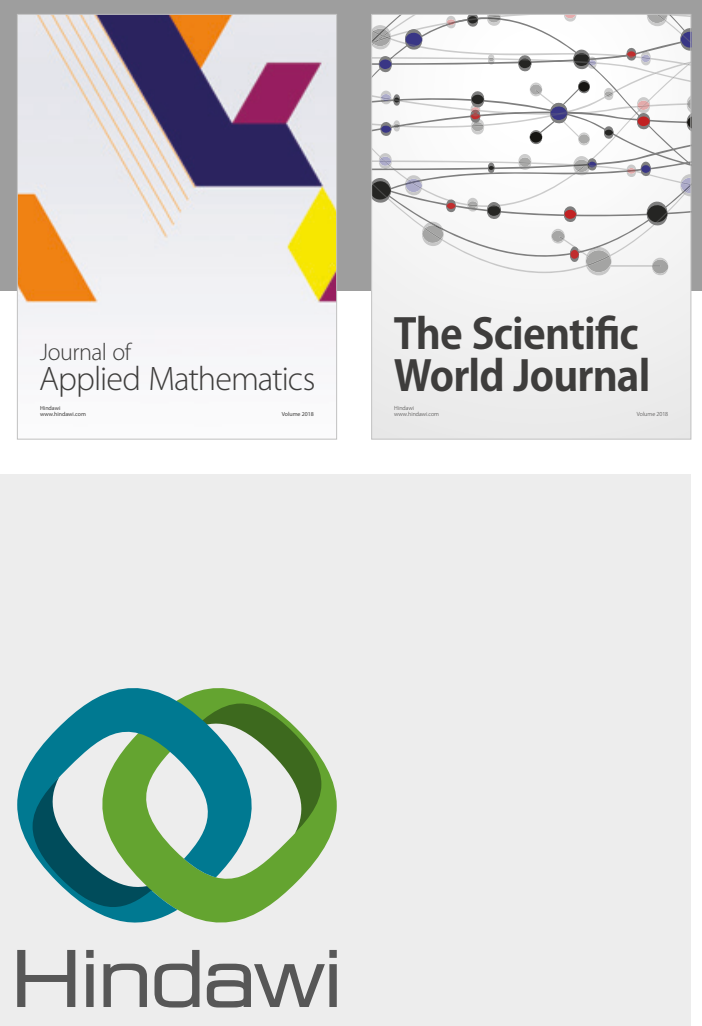

Submit your manuscripts at

www.hindawi.com

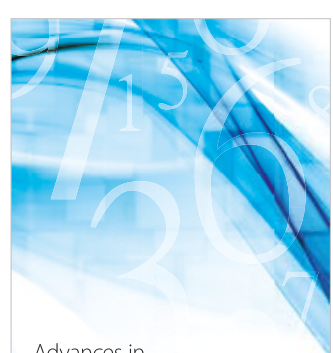

Advances in
Numerical Analysis
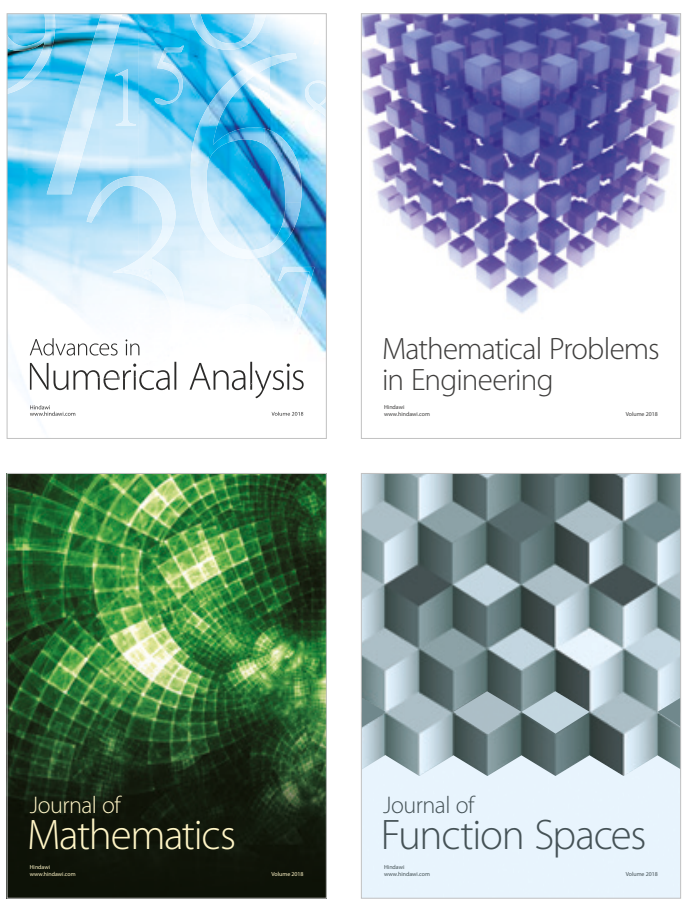

Mathematical Problems in Engineering

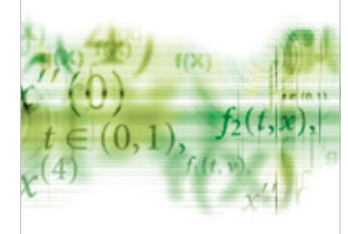

International Journal of

Differential Equations

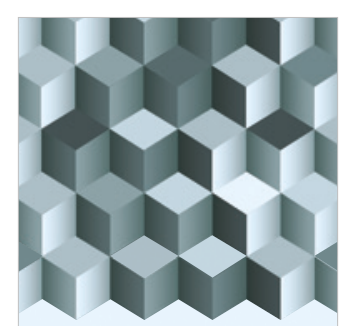

Journal of

Function Spaces
The Scientific

World Journal

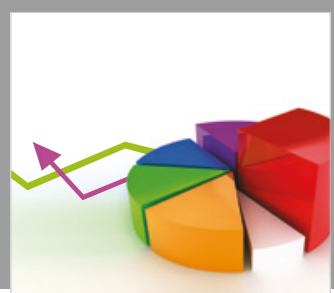

Journal of

Probability and Statistics
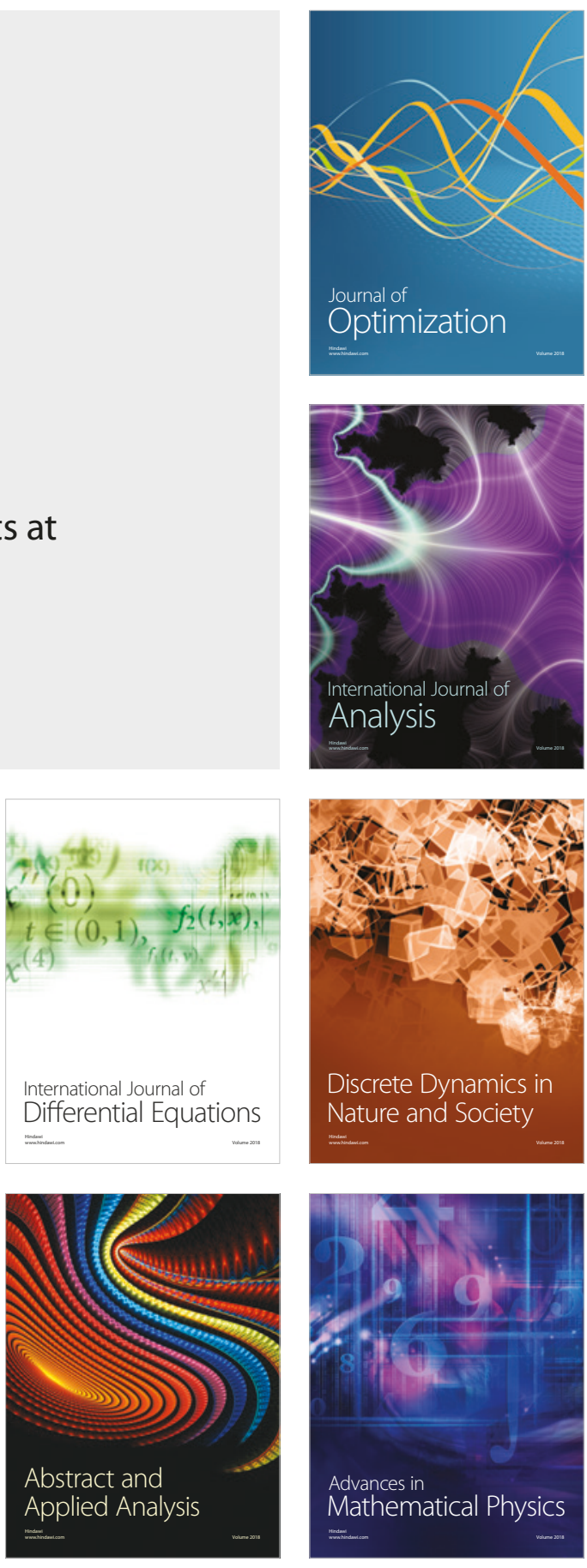